\title{
Spatial mapping of ground-based observations of total ozone
}

\author{
K.-L. Chang ${ }^{1}$, S. Guillas ${ }^{1}$, and V. E. Fioletov ${ }^{2}$ \\ ${ }^{1}$ Department of Statistical Science, University College London, London, UK \\ ${ }^{2}$ Environment Canada, Toronto, Ontario, Canada \\ Correspondence to: K.-L. Chang (ucakkac@ucl.ac.uk) \\ Received: 26 January 2015 - Published in Atmos. Meas. Tech. Discuss.: 22 April 2015 \\ Revised: 29 September 2015 - Accepted: 7 October 2015 - Published: 23 October 2015
}

\begin{abstract}
Total column ozone variations estimated using ground-based stations provide important independent source of information in addition to satellite-based estimates. This estimation has been vigorously challenged by data inhomogeneity in time and by the irregularity of the spatial distribution of stations, as well as by interruptions in observation records. Furthermore, some stations have calibration issues and thus observations may drift. In this paper we compare the spatial interpolation of ozone levels using the novel stochastic partial differential equation (SPDE) approach with the covariance-based kriging. We show how these new spatial predictions are more accurate, less uncertain and more robust. We construct long-term zonal means to investigate the robustness against the absence of measurements at some stations as well as instruments drifts. We conclude that time series analyzes can benefit from the SPDE approach compared to the covariance-based kriging when stations are missing, but the positive impact of the technique is less pronounced in the case of drifts.
\end{abstract}

\section{Introduction}

The ground-based total column ozone data set is based on Dobson and Brewer spectrophotometer and filter ozonometer observations available from the World Ozone and UV Data Centre (WOUDC) (http://www.woudc.org/). Large longitudinal inhomogeneities in the global ozone distribution and limited spatial coverage of the ground-based network make it difficult to estimate zonal and global total ozone values from station observations directly (Fioletov et al., 2002). The Total Column Ozone (TCO) data set is comprised of the ozone observations from the set of ground-based stations worldwide. Most of those stations are located on land in the Northern
Hemisphere, and relatively few stations are over the Southern Hemisphere and oceans. Therefore the spatial distribution of ground-based stations is highly irregular. In addition, durations of operations for each station are different. One of the major difficulties in assessing long-term global total ozone variations is thus data inhomogeneity. Indeed recalibration of ground-based instruments, or interruptions in observation records result in data sets which may have systematic errors that change with time (Fioletov et al., 2002, 2008).

The TCO data set and corresponding satellite measurements have also been widely discussed in the statistics literature. Some authors have noticed space-time asymmetry in ozone data (Stein, 2005, 2007; Jun and Stein, 2007, 2008). The other important feature of TCO data is that the spatial dependence of ozone distributions varies strongly with latitude and weakly with longitude, so that homogeneous models (invariant to all rotations) are clearly unsuitable (Stein, 2007). This is why Jun and Stein (2007) assume that the spatial process driving the TCO data is an axially symmetric process whose first two moments are invariant to rotations about the Earth's axis, and constructed space-time covariance functions on the sphere $\times$ time that are weakly stationary with respect to longitude and time for fixed values of latitude. Jun and Stein (2008) further used linear combinations of Legendre polynomials to represent the coefficients of partial differential operators in the covariance functions. These covariance functions produce covariance matrices that are neither of low rank nor sparse for irregularly distributed observations, as it is the case with ground-based stations. Hence, likelihood calculations can thus be difficult in that situation, and we will not follow this approach.

The aim of this article is to apply a new technique, the stochastic partial differential equation (SPDE) approach in spatial statistics (Lindgren et al., 2011) in order to best evalu- 
ate total column ozone spatially from ground-based stations. The SPDE approach has already been applied to regularly spaced ozone satellite data by Bolin and Lindgren (2011) but not to ground-level stations, where gains in accuracy are potentially larger due to the gaps in coverage. Furthermore, we quantify the impact of the improvement of these spatial estimations on the computations of time series over various regions. Finally, the SPDE and covariance-based approaches are also used to calculate monthly zonal mean total ozone values and compare them with zonal means calculated from ground-based data and available from the WOUDC (Bojkov and Fioletov, 1995; Fioletov et al., 2002).

Section 2 gives a brief introduction to the theoretical framework of the SPDE technique, a basic description of the covariance-based kriging and related model selection and diagnostic techniques. Section 3 describes the spatial analysis using TCO data from WOUDC on a monthly, seasonal and annual basis. Furthermore, the estimated results of SPDE and covariance-based kriging are compared with the Total Ozone Mapping Spectrometer (TOMS) satellite data to examine which method yields approximations closer to satellite data. Finally, the long-term zonal mean trends enable us to conduct a sensitivity analysis by removing stations at random and by introducing long-term drifts at some groundbased stations.

\section{Methods}

Our main problem is to estimate ozone values at places where it is not observed. Models in spatial statistics that enable this task are usually specified through the covariance function of the latent field. Indeed, in order to assess uncertainties in the spatial interpolation with global coverage, we cannot build models only for the discretely located observations, we need to build an approximation of the entire underlying stochastic process defined on the sphere. We consider statistical models for which the unknown functions are assumed to be realizations of a Gaussian random spatial process. The standard fitting approach, covariance-based kriging, spatially interpolates values as linear combinations of the original observations, and this constitutes the spatial predictor. Not only large data sets can be computationally demanding for a kriging predictor but covariance-based models also struggle to take into consideration in general nonstationarity (i.e., when physical spatial correlations are different across regions) due to the fixed underlying covariance structure. Recently, a different computational approach (for identical underlying spatial covariance models) was introduced by Lindgren et al. (2011), in which random fields are expressed as a weak solution to an SPDE, with explicit links between the parameters of the SPDE and the covariance structure. This approach can deal with large spatial data sets and naturally account for nonstationarity. We review below some of the recent development on the covariance structure modeling on the sphere, with a particular focus on SPDE and covariancebased kriging. Computational implementations of SPDE and covariance-based kriging, with mathematical details, are relegated to Appendix A.

The Matérn covariance function is an advanced covariance structure used to model dependence of spatial data on the plane. On the sphere, Guinness and Fuentes (2013) show that the kriging prediction using the Matérn function with chordal distance outperforms many other types of isotropic covariance functions, both in terms of accuracy and quantification of uncertainty. The shape parameter $\nu$, scale parameter $\kappa$ and the marginal precision $\tau^{2}$ parameterize it:

$$
C(\boldsymbol{h})=\frac{2^{1-v}}{(4 \pi)^{d / 2} \Gamma(v+d / 2) \kappa^{2 v} \tau^{2}}(\kappa\|\boldsymbol{h}\|)^{v} K_{v}(\kappa\|\boldsymbol{h}\|),
$$

where $\boldsymbol{h} \in \mathbb{R}^{d}$ denotes the difference between any two locations $\boldsymbol{s}$ and $\boldsymbol{s}^{\prime}: \boldsymbol{h}=\boldsymbol{s}-\boldsymbol{s}^{\prime}$, and $K_{v}$ is a modified Bessel function of the second kind of order $v>0$.

\subsection{SPDE approach}

Let $X(\mathbf{s})$ be the latent field of ozone measurements $Y(\mathbf{s})$ under observation errors $\epsilon(\mathbf{s})$. Lindgren et al. (2011) use the fact that a random process $X(\mathbf{s})$ on $\mathbb{R}^{d}$ with a Matérn covariance function (Eq. 1) is the stationary solution to the SPDE:

$\left(\kappa^{2}-\Delta\right)^{\alpha / 2} \tau X(\mathbf{s})=\mathcal{W}(\mathbf{s})$,

where $\mathcal{W}(\mathbf{s})$ is Gaussian white noise, and $\Delta$ is the Laplace operator. The regularity (or smoothness) parameter $v$ essentially determines the order of differentiability of the fields. The link between the Matérn covariance (Eq. 1) and the SPDE formulation (Eq. 2) is given by $\alpha=v+d / 2$, which makes explicit the relationship between dimension and regularity for fixed $\alpha$. Unlike the covariance-based model, the SPDE approach can be easily manipulated on manifolds. On more general manifolds than $\mathbb{R}^{d}$, the direct Matérn representation is not easy to implement, but the SPDE formulation provides a natural generalization, and the $v$ parameter will keep its meaning as the quantitative measure of regularity. Instead of defining Matérn fields by the covariance function on these manifolds, Lindgren et al. (2011) used the solution of the SPDE as a definition, and it is much easier and flexible to do so. This definition is valid not only on $\mathbb{R}^{d}$ but also on general smooth manifolds, such as the sphere. The SPDE approach allows $\kappa$ and $\tau$ to vary with location:

$\left(\kappa^{2}(\mathbf{s})-\Delta\right)^{\alpha / 2} \tau(\mathbf{s}) X(\mathbf{s})=\mathcal{W}(\mathbf{s})$,

where $\kappa(\mathbf{s})$ and $\tau(\mathbf{s})$ are estimated by expanding them in a basis of a function space such as the spherical harmonic basis. We estimate the SPDE approach parameters and supply uncertainty information about the surfaces by using the integrated nested Laplacian approximations (INLA) framework, available as an R package (http://www.r-inla.org/). For latent Gaussian Markov random fields used to efficiently solve 
SPDEs on triangulations, INLA provides good approximations of posterior densities at a fraction of the cost of Markov chain Monte Carlo. Note that for models with Gaussian data, the calculated densities are for practical purposes exact.

\subsection{Covariance-based approach}

For locations on spherical domain, $\mathbf{s}_{i}=\left(\mathbf{L}_{i}, \mathbf{l}_{i}\right)$, let $Y\left(\mathbf{s}_{i}\right)$ denote the ozone measured at station $i$. Then the kriging representation can be assumed additive with a polynomial model for the spatial trend (universal kriging):

$Y\left(\mathbf{s}_{i}\right)=P\left(\mathbf{s}_{i}\right)+Z\left(\mathbf{s}_{i}\right)+e_{i}$,

where $P$ is a polynomial which is the fixed part of the model, $Z$ is a zero mean, Gaussian stochastic process with an unknown covariance function $K$ and $e_{i}$ are i.i.d. normal errors. The estimated latent field is then the best linear unbiased estimator (BLUE) of $P(\mathbf{s})+Z(\mathbf{s})$ given the observed data. Note that the Gaussian process in the spatial model, $Z(s)$, can be defined as a realization of the process $X(s)$ from the previous section.

For the covariance-based approach, the hurdle that we are facing is that we have to define a valid (but flexible enough) covariance model and, furthermore, compared to data on the plane, we must employ a distance on the sphere. Two distances are commonly considered. The chordal distance between the two points $\left(\mathbf{L}_{1}, \mathbf{l}_{1}\right)$ and $\left(\mathbf{L}_{2}, \mathbf{l}_{2}\right)$ on the sphere is given by

$\operatorname{ch}\left(\mathbf{L}_{1}, \mathbf{L}_{2}, \mathbf{l}_{1}-\mathbf{l}_{2}\right)=$

$2 r\left(\sin ^{2}\left(\frac{\mathbf{L}_{1}-\mathbf{L}_{2}}{2}\right)+\cos \mathbf{L}_{1} \cos \mathbf{L}_{2} \sin ^{2}\left(\frac{\mathbf{l}_{1}-\mathbf{l}_{2}}{2}\right)\right)^{1 / 2}$,

where $r$ denotes the Earth's radius. The more physically intuitive great circle distance between the two locations is $\operatorname{gc}\left(\mathbf{L}_{1}, \mathbf{L}_{2}, \mathbf{l}_{1}-\mathbf{l}_{2}\right)=2 r \arcsin \left\{\operatorname{ch}\left(\mathbf{L}_{1}, \mathbf{L}_{2}, \mathbf{l}_{1}-\mathbf{l}_{2}\right)\right\}$. However, Gneiting (2013) pointed out that using the great circle distance in the original Matern covariance function (Eq. 1) would not work, as it may not yield a valid positive definite covariance function. Therefore in this study we use the chordal distance for covariance-based kriging. The main advantage of using the chordal distance is that it is well defined on spherical domains, as it restricts positive definite covariance functions on $\mathbb{R}^{3}$ to $\mathbb{S}^{2}$ (Jun and Stein, 2007). For the ozone data, we specify the Matern covariance function defined in Eq. (1) in the covariance-based approach in order to compare the performance with the SPDE approach for exactly the same covariance function, whereas the model parameters are optimized according to different techniques. The relevant model diagnostic and selection criteria are described in Appendix B. Note that the smoothness parameter $v$ is allowed to be selected in the covariance-based kriging, whereas we fix it at $v=1$ in the SPDE approach.

\section{Mapping accuracy}

In this section, we produce statistical estimates of monthly ozone maps, using TCO data from WOUDC. We consider TCO data in January 2000 as an illustration, which contain 150 ground-based ozone observations around the world. All ozone values in this article are in Dobson units (DU). We first choose the model setups for both SPDE and covariancebased approaches below.

With the SPDE approach, as the smoothness parameter $v$ is defined through the relationship $\alpha=v+d / 2$ (see Appendix A1), we only need to choose the basis expansion order to represent $\kappa$ and $\tau$. To choose the best maximal order of the spherical harmonic basis, we fitted models with different maximal orders of spherical harmonics for the expansions of $\kappa$ and $\tau$ in order to estimate them thereafter (the default formulation in the R-INLA package). The best fitted model is for a spherical harmonics basis with maximal order 3 since it yielded the lowest generalized cross-validation (GCV) standard deviation (SD) computed in Eq. (B2), which yields a total of nine parameters to be estimated (four parameters for the expansions of $\kappa$ and $\tau$ and one parameter for the variance). For the covariance-based approach, we need to choose the smoothness parameter in the Matérn covariance function before estimating the univariate quantities $\kappa$ and $\tau^{2}$. The same $\sigma_{\mathrm{GCV}}$ criterion is used to evaluate the model performance. We fit a wide range of values for the smoothing parameter, and $v=20$ minimizes the $\sigma_{\mathrm{GCV}}$ for these ozone data.

To compare the performance of the SPDE approach with the covariance-based kriging, the same $\sigma_{\mathrm{GCV}}$ criterion is used as it balances well predictive power vs. overfitting across methods. We first estimate the optimized model parameters, i.e., $\kappa$ and $\tau$ in a SPDE and $v$ in a covariancebased model, by the same generalized cross-validation criterion (with different computational methods, the estimated method for the chordal distance covariance model is maximum likelihood estimates), then compare the uncertainty of spatial estimation over the surface. For the prior specifications of the SPDE approach, $\kappa$ and $\tau$ follow log normal priors by default: precision (theta.prior.prec) $=0.1$, median for $\tau$ (prior.variance.nominal) $=1$ and median for $\kappa$ (prior.range.nominal) depends on the mesh. We use a regression basis of spherical harmonics for both of these parameters; therefore by default the coefficients follow the log normal priors. We do not change the R-INLA default prior settings throughout the analysis.

In order to achieve a better estimation, the monthly mean "norms" (Bojkov and Fioletov, 1995) (or total ozone "climatology") are calculated for each station and each month of the year over the whole period and then subtracted from the data. The norms are used as first approximations to remove the general spatial trend. For each station and for each month, spatial interpolations through SPDE and covariancebased approaches were performed to these deviations. 


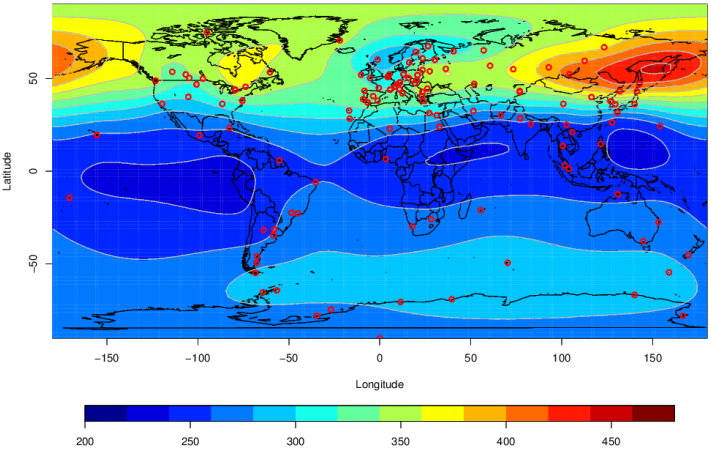

(a) SPDE mean

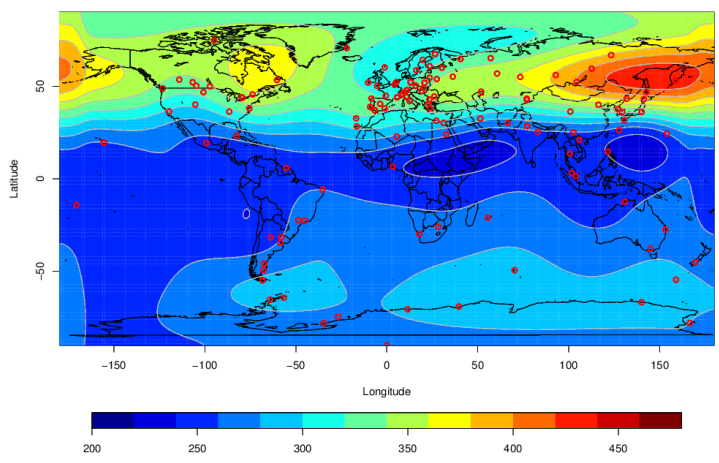

(c) CBK mean

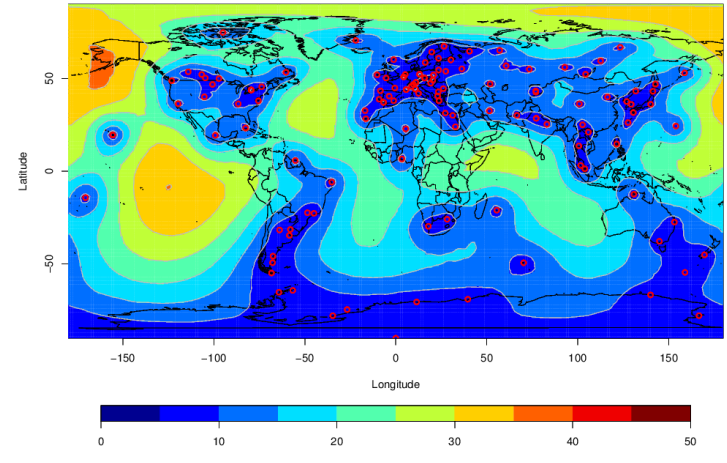

(b) SPDE SD

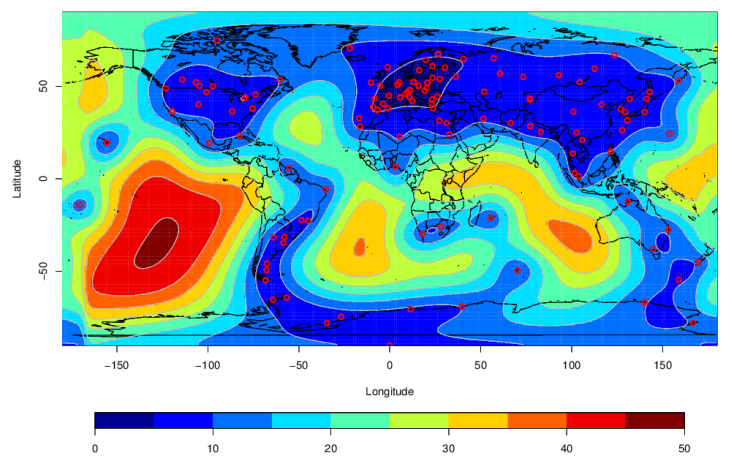

(d) CBK SD

Figure 1. Surface predicted ozone (DU) mean and SD for SPDE (strategy D) and CBK (strategy B) from January 2000. The red points indicate the locations of stations.

Table 1. Specifications of the different strategies in the spatial estimations.

\begin{tabular}{ll}
\hline Strategy & Descriptions \\
\hline A & A covariance-based model with $v=1$ \\
B & A covariance-based model with $\nu=20$ \\
C & An SPDE-based model with stationary covariance \\
& $\begin{array}{l}\text { parameters (i.e., } \kappa \text { and } \tau \text { are unknown constants) } \\
\text { D }\end{array}$ \\
& covariance parameters \\
\hline
\end{tabular}

We now compare the results estimated by four strategies described in Table 1. We start an illustration to 6 years of monthly observations. The results of the analysis of ozone data averaged from 2000 to 2005 are shown in Table 2. The averaged number of available stations is denoted by $\bar{n}$, and the residual sum of square (RSS) indicates residuals sum of square, which is defined in Eq. (B1). The SPDE approach (strategies $\mathrm{C}$ and $\mathrm{D}$ ) provides a better fit than the covariancebased kriging (strategies A and B) for all months. The effective degree of freedom $n_{\text {eff }}$ is higher in the SPDE approach as it is more complex than covariance-based kriging. Higher effective degrees of freedom means smaller values in the $\sigma_{\mathrm{GCV}}$ denominator $\left(n-n_{\mathrm{eff}}\right)$ and thus higher values for $\sigma_{\mathrm{GCV}}$ to account for overparametrization. Nevertheless, $\sigma_{\mathrm{GCV}}$ values for the SPDE approach are still all much lower than for covariance-based kriging in all cases. This means that the RSS in the SPDE approach is drastically smaller than the RSS for covariance-based kriging. Thus the SPDE approach supplies a much better fit to the true ozone observation. Note that the covariance-based model is computing a weighted average of the neighborhood values around the location, while the SPDE model is constructed through a triangular mesh. The mesh can be more adaptive and flexible to irregularly distributed observations. In addition, with spatially varying $\kappa$ and $\tau$, the table indicates that the results could be improved further by applying a nonstationary extension.

Table 2 also reports regional RSSs by dividing the Earth into the Northern Hemisphere $\left(>30^{\circ} \mathrm{N}\right)$, tropics $\left(30^{\circ} \mathrm{S}-\right.$ $\left.30^{\circ} \mathrm{N}\right)$ and Southern Hemisphere $\left(<30^{\circ} \mathrm{S}\right)$. In general, over half of the ground-based stations are located on the Northern Hemisphere, which gives rise to a higher RSS with respect to other regions as the RSSs are not normalized by the number of observations. RSSs estimated by SPDE and covariancebased kriging show similar patterns across months and across 
Table 2. Comparison of the generalized cross-validation error $\left(\sigma_{\mathrm{GCV}}\right)$ and the residual sum of square (RSS) for different strategies averaged of 2000-2005 by month. Statistical summaries for Northern Hemisphere (NH), tropics and Southern Hemisphere (SH).

\begin{tabular}{|c|c|c|c|c|c|c|c|c|c|c|c|c|c|}
\hline Global statistics & Number of obs. & $\begin{array}{r}\text { Jan } \\
135.33\end{array}$ & $\begin{array}{r}\text { Feb } \\
144.33\end{array}$ & $\begin{array}{r}\text { Mar } \\
146.17\end{array}$ & $\begin{array}{r}\text { Apr } \\
146.00\end{array}$ & $\begin{array}{r}\text { May } \\
142.50\end{array}$ & $\begin{array}{r}\text { Jun } \\
143.33\end{array}$ & $\begin{array}{r}\text { Jul } \\
143.00\end{array}$ & $\begin{array}{r}\text { Aug } \\
145.33\end{array}$ & $\begin{array}{r}\text { Sep } \\
146.00\end{array}$ & $\begin{array}{r}\text { Oct } \\
142.67\end{array}$ & $\begin{array}{r}\text { Nov } \\
140.17\end{array}$ & $\begin{array}{r}\text { Dec } \\
129.50\end{array}$ \\
\hline \multirow[t]{3}{*}{ A } & $n_{\text {eff }}$ & 16.00 & 20.92 & 26.84 & 17.50 & 28.23 & 22.96 & 11.20 & 31.67 & 53.11 & 60.29 & 30.09 & 19.67 \\
\hline & $\sigma_{\mathrm{GCV}}$ & 16.62 & 17.51 & 14.97 & 13.29 & 10.83 & 10.23 & 10.35 & 11.20 & 13.19 & 12.67 & 17.17 & 15.58 \\
\hline & RSS & 34584 & 39599 & 28017 & 23405 & 13884 & 13333 & 14300 & 14688 & 19312 & 13379 & 34470 & 28416 \\
\hline \multirow[t]{3}{*}{ B } & $n_{\text {eff }}$ & 16.89 & 25.69 & 30.51 & 19.57 & 24.14 & 15.46 & 14.39 & 29.63 & 36.93 & 38.24 & 28.86 & 20.67 \\
\hline & $\sigma_{\mathrm{GCV}}$ & 15.10 & 14.05 & 12.11 & 12.45 & 10.33 & 10.54 & 9.87 & 9.75 & 9.68 & 9.53 & 12.35 & 12.71 \\
\hline & RSS & 28535 & 25611 & 18323 & 20711 & 13258 & 14770 & 12637 & 11479 & 10654 & 9645 & 17550 & 19129 \\
\hline \multirow[t]{3}{*}{$\mathrm{C}$} & $n_{\text {eff }}$ & 70.33 & 60.76 & 81.23 & 59.31 & 64.87 & 76.23 & 48.70 & 54.63 & 65.13 & 75.41 & 72.09 & 51.12 \\
\hline & $\sigma_{\mathrm{GCV}}$ & 11.89 & 10.33 & 8.39 & 9.24 & 6.06 & 6.77 & 7.78 & 7.61 & 6.71 & 6.15 & 9.45 & 8.91 \\
\hline & RSS & 12263 & 10085 & 5394 & 9600 & 3428 & 4283 & 6224 & 6351 & 4400 & 2600 & 6924 & 6897 \\
\hline \multirow[t]{3}{*}{ D } & $n_{\text {eff }}$ & 67.75 & 59.12 & 73.08 & 61.01 & 72.01 & 84.44 & 60.14 & 64.71 & 73.67 & 75.21 & 72.00 & 62.97 \\
\hline & $\sigma_{\mathrm{GCV}}$ & 8.60 & 9.90 & 7.90 & 9.18 & 6.27 & 6.80 & 7.22 & 6.85 & 6.62 & 5.75 & 8.84 & 7.90 \\
\hline & RSS & 6148 & 9431 & 5312 & 8664 & 3359 & 3865 & 4326 & 4845 & 4179 & 2285 & 5924 & 4805 \\
\hline \multirow[t]{2}{*}{ RSS by region } & & Jan & Feb & Mar & Apr & May & Jun & Jul & Aug & Sep & Oct & Nov & Dec \\
\hline & Number of obs. & 89.50 & 98.50 & 101.67 & 104.00 & 102.50 & 103.83 & 103.00 & 103.00 & 102.67 & 98.50 & 95.50 & 84.67 \\
\hline \multirow[t]{5}{*}{ NH } & A & 29531 & 34011 & 23399 & 17207 & 9981 & 9692 & 8844 & 6933 & 6118 & 4159 & 16117 & 22115 \\
\hline & B & 26035 & 22234 & 15507 & 16144 & 10498 & 11288 & 8435 & 7238 & 7179 & 6748 & 13035 & 16690 \\
\hline & C & 11543 & 9248 & 4895 & 8002 & 2932 & 3415 & 4467 & 4519 & 3490 & 2011 & 5649 & 6082 \\
\hline & $\mathrm{D}$ & 4119 & 7240 & 4183 & 6696 & 2880 & 3328 & 3384 & 3903 & 3709 & 1914 & 5104 & 3301 \\
\hline & Number of obs. & 27.83 & 27.33 & 26.33 & 26.33 & 26.50 & 26.67 & 27.33 & 27.33 & 26.83 & 26.67 & 27.00 & 27.17 \\
\hline \multirow[t]{5}{*}{ Tropics } & A & 3706 & 3666 & 3461 & 4082 & 1758 & 1671 & 2766 & 2253 & 2898 & 2286 & 4123 & 3008 \\
\hline & B & 1778 & 2182 & 2120 & 2988 & 1254 & 1632 & 2264 & 1228 & 957 & 1042 & 1360 & 1411 \\
\hline & $\mathrm{C}$ & 413 & 489 & 367 & 1095 & 219 & 280 & 830 & 489 & 238 & 192 & 397 & 454 \\
\hline & $\mathrm{D}$ & 1148 & 1438 & 688 & 1142 & 331 & 368 & 646 & 441 & 171 & 120 & 309 & 990 \\
\hline & Number of obs. & 18.00 & 18.50 & 18.17 & 15.67 & 13.50 & 12.83 & 12.67 & 15.00 & 16.50 & 17.50 & 17.67 & 17.67 \\
\hline \multirow[t]{4}{*}{$\mathrm{SH}$} & A & 1347 & 1922 & 1157 & 2116 & 2144 & 19670 & 2689 & 5502 & 10296 & 6933 & 14230 & 3293 \\
\hline & B & 722 & 1195 & 696 & 1580 & 1505 & 1850 & 1938 & 3012 & 2518 & 1855 & 3155 & 1028 \\
\hline & C & 306 & 348 & 162 & 504 & 277 & 588 & 927 & 1343 & 672 & 397 & 878 & 361 \\
\hline & D & 882 & 754 & 442 & 827 & 148 & 169 & 295 & 501 & 299 & 251 & 510 & 515 \\
\hline
\end{tabular}

regions. Lower estimation errors can be found in AugustOctober and higher errors occur in December-February.

Figure 1 shows the predicted mean and SD ozone maps by strategies B and D on January 2000. The spatial distributions of ozone means are similar for SPDE and covariancebased kriging in the Northern Hemisphere, but there are differences in the Southern Hemisphere. These differences arise from the asymmetry of available stations in the two hemispheres. The spatial distributions of the SD present similar general patterns for the two techniques. The uncertainties are higher where with fewer stations are available, see the large uncertainty distribution over the South Pacific Ocean. SDs of SPDE predictions are much smaller than the SDs of the covariance-based kriging predictions, especially where fewer observations are available (e.g., mid-Atlantic and South Pacific), but are larger near the North Pole; this may be due to covariance-based kriging underestimating its own uncertainty.

\subsection{Seasonal and annual effects}

Seasonal ozone data are obtained by averaging the corresponding monthly data (but all months of every season must be available to create such seasonal averages). Table 3 shows the seasonal results for different strategies over the years 2000-2005. Their respective highest errors are in December-January-February (DJF) for strategies A, B and $\mathrm{C}$ and in June-July-August (JJA) for strategy D. The results are hardly different in March-April-May (MAM) and September-October-November (SON) for strategies C and $\mathrm{D}$, while a significant improvement can be achieved in DJF by applying a nonstationary SPDE model. Moreover, the values of $\sigma_{\mathrm{GCV}}$ and RSS from this seasonal analysis are smaller than the corresponding analysis for each month of the associated season, both for SPDE and covariance-based kriging, and are also closer across the two competing techniques due to additional averaging smoothing out the gains in accuracy. Nevertheless the SPDE approach still provides a better fit than covariance-based kriging in all seasons. Figures 2 and 3 show the seasonal ozone maps by strategy $\mathrm{D}$ of means and 


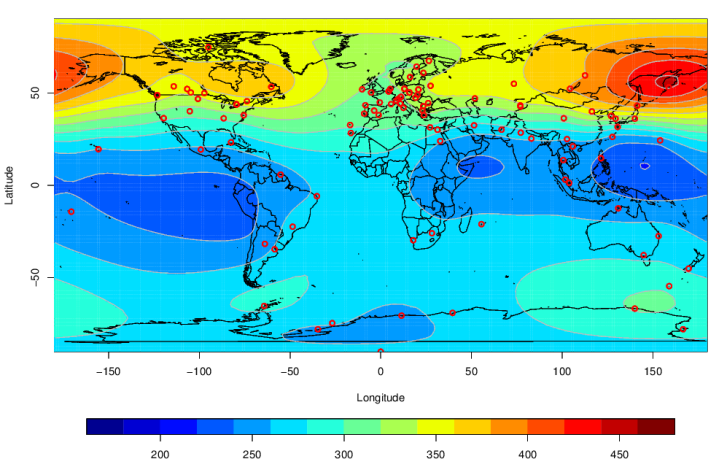

(a) DJF

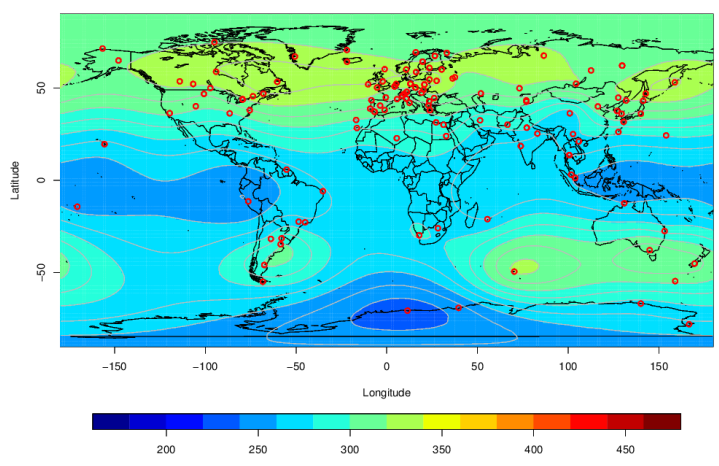

(c) JJA

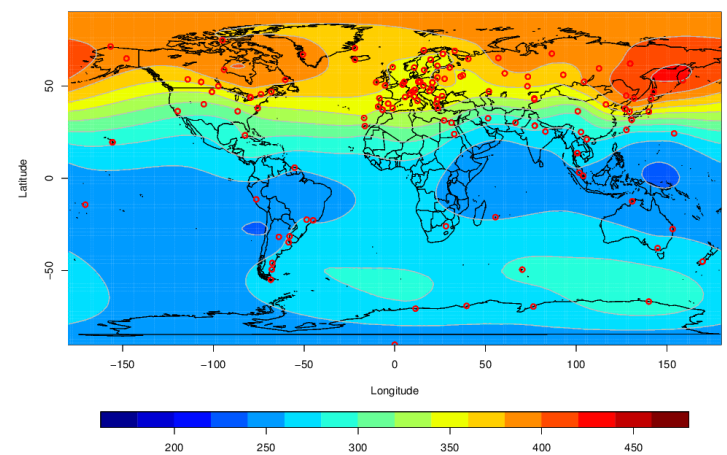

(b) MAM

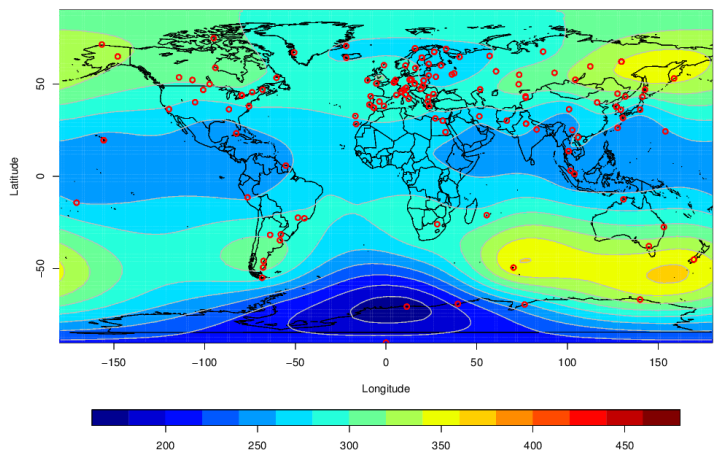

(d) $\mathrm{SON}$

Figure 2. Surface predicted ozone (DU) mean from SPDE approach (strategy D) by season from 2000.

Table 3. Generalized cross-validation error $\left(\sigma_{\mathrm{GCV}}\right)$ and residual sum of square (RSS) for different strategies averaged over 20002005 by season.

\begin{tabular}{llrrrrr}
\hline & Season & DJF & MAM & JJA & SON & Average \\
& $\bar{n}$ & 117.17 & 132.83 & 132.17 & 130.67 & 128.21 \\
\hline $\mathrm{A}$ & $n_{\text {eff }}$ & 13.45 & 21.66 & 13.01 & 52.06 & 25.04 \\
& $\sigma_{\mathrm{GCV}}$ & 11.95 & 9.96 & 8.65 & 11.23 & 10.45 \\
& $\mathrm{RSS}$ & 16194 & 11513 & 8987 & 10178 & 11718 \\
\hline $\mathrm{B}$ & $n_{\mathrm{eff}}$ & 18.73 & 20.68 & 11.53 & 32.77 & 20.93 \\
& $\sigma_{\mathrm{GCV}}$ & 9.47 & 9.20 & 8.39 & 8.19 & 8.81 \\
& $\mathrm{RSS}$ & 10274 & 9990 & 8526 & 6783 & 8893 \\
\hline $\mathrm{C}$ & $n_{\mathrm{eff}}$ & 48.10 & 60.41 & 50.25 & 75.00 & 58.44 \\
& $\sigma_{\mathrm{GCV}}$ & 7.72 & 5.86 & 6.41 & 6.47 & 6.46 \\
& $\mathrm{RSS}$ & 4661 & 3120 & 4140 & 2272 & 3548 \\
\hline $\mathrm{D}$ & $n_{\mathrm{eff}}$ & 52.82 & 64.43 & 58.06 & 77.07 & 63.09 \\
& $\sigma_{\mathrm{GCV}}$ & 6.55 & 6.00 & 6.13 & 5.82 & 6.12 \\
& $\mathrm{RSS}$ & 2695 & 3074 & 3609 & 2210 & 2897 \\
\hline
\end{tabular}

$\mathrm{SD}$, respectively. The maps for SD again reveal the higher estimated error in regions without stations.

The annual ozone data are obtained by creating an annual average, which also means that stations with record in- terruptions are not used. Therefore fewer stations are available for this exercise. To see the improvement of the annualbased analysis over seasonally and monthly analyses, Table 4 shows the annual averaged results by month and seasonally, and the results directly obtained by doing the analysis on the annual mean. Although there are fewer stations in annual-based and seasonal-based data than in monthly data, the errors are lower than for monthly data over the years for all strategies and the results directly obtained from annual means yield even lower RSS and $\sigma_{\mathrm{GCV}}$ than the results averaged by seasons due to smooth variation.

\subsection{Comparison with satellite data}

In this section, we assess the match between satellite observations and spatial predictions based on ground-level stations. The TOMS data on monthly averages are obtained from the NASA website (http://ozoneaq.gsfc.nasa. gov/), where we collected the Earth Probe (25 July 199631 December 2005) satellite data with grid $1^{\circ} \times 1.25^{\circ}$. We calculate the differences over all grid cells and summarize it by the root mean square error (RMSE). Let $\hat{y}_{i}$ be the estimated result from the SPDE or covariance-based kriging on grid $i$, and let $y_{i}^{\mathrm{s}}$ denote the satellite value on grid $i$, then the 


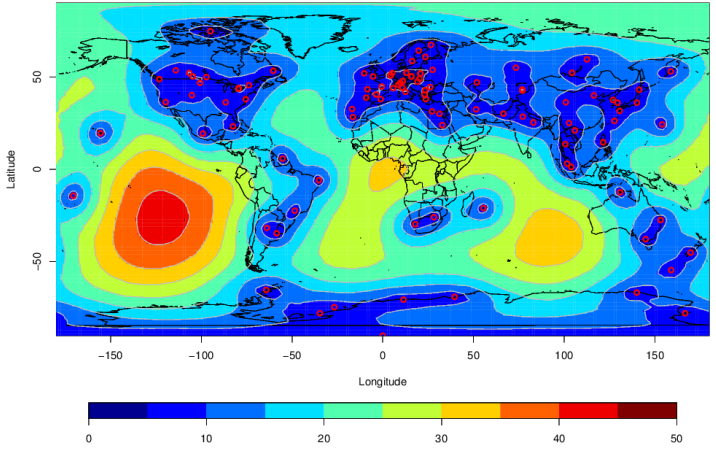

(a) DJF

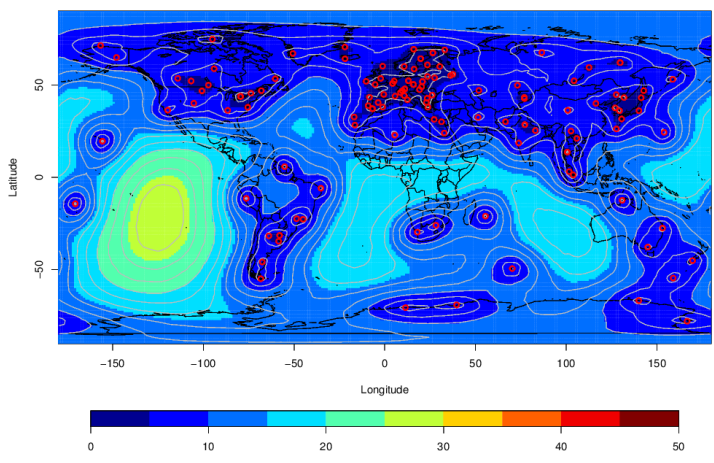

(c) JJA

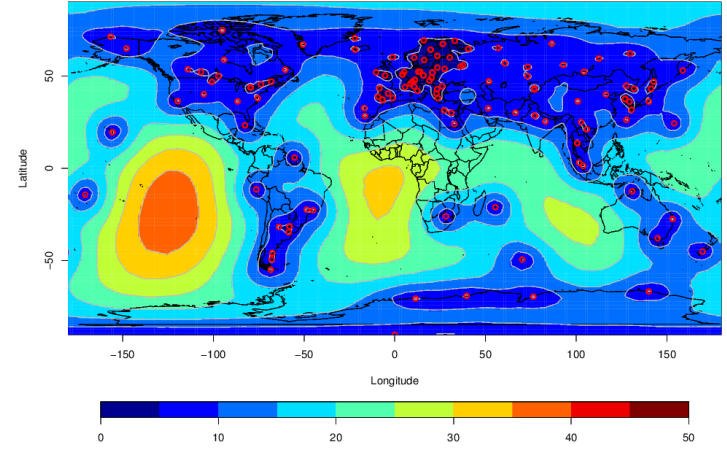

(b) MAM

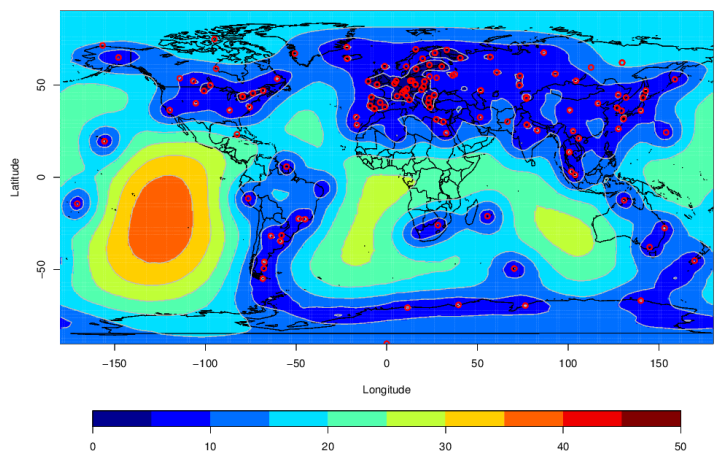

(d) $\mathrm{SON}$

Figure 3. Surface predicted ozone (DU) standard deviation from SPDE approach (strategy D) by season from 2000.

(normalized) RMSE is given by

$\operatorname{RMSE}=\sqrt{\frac{\sum_{i=1}^{n}\left(\hat{y}_{i}-y_{i}^{\mathrm{s}}\right)^{2}}{n}}$,

where $n=180 \times 288$ is total number of grid cells. However, satellite data are unavailable over high latitudes in DJF and MAM and over low latitudes in JJA and SON. Therefore we restrict the calculations of RMSEs between $60^{\circ} \mathrm{S}$ and $60^{\circ} \mathrm{N}$.

From this stage we only compare the results between a nonstationary SPDE-based model and a covariance-based model with $v=20$. Monthly comparisons over 2000-2005 are shown in Table 5. Ozone surfaces predicted by the SPDE approach are closer to the satellite data than the predictions from covariance-based kriging over all months. The highest improvement of SPDE over covariance-based kriging is $77.15 \%$ in September and the lowest is $33.60 \%$ in May. Also, in contrast with relatively unstable monthly predictions by covariance-based kriging, SPDE shows more consistency in predictions of monthly ozone variation.

Figures 4 and 5 map the differences of surface predictions of covariance-based and SPDE methods with respect to satellite data over $60^{\circ} \mathrm{S}-60^{\circ} \mathrm{N}$ on January, April, July and October 2000. The differences in October turn out to be much larger than in other months, and therefore a different scale is used. These maps indicate the overestimation (red) and underestimation (blue) with respect to satellite data. Similar patterns in deviations are revealed for both techniques, but SPDE displays less magnitude in the deviations than covariance-based kriging. One noticeable feature is that the pattern of deviation from satellite data is strongly related to the distribution of ground-based stations: for instance, the covariance-based kriging predicted surfaces tend to underestimate the values over the South Pacific Ocean, where few stations are available. The surface predictions by SPDE achieve a clear improvement in predictions compared to covariance-based kriging over areas with less stations, especially in January and October.

The seasonal predicted total ozone is obtained by averaging the corresponding monthly means. We excluded stations that have interruptions in their records. Therefore fewer observations are used to predict seasonal means. The RMSEs between predicted surface and satellite data are presented in Table 6. In general, seasonal maps should agree better with satellite-based maps than monthly maps. However, fewer observations are used in seasonal predictions and that may trigger high RMSEs in the covariance-based kriging es- 


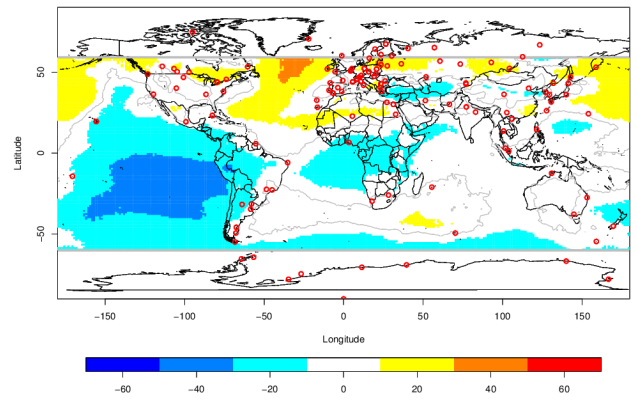

(a) SPDE (January)

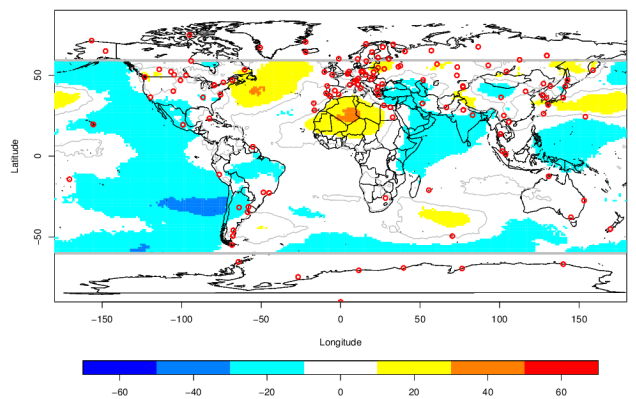

(c) SPDE (April)

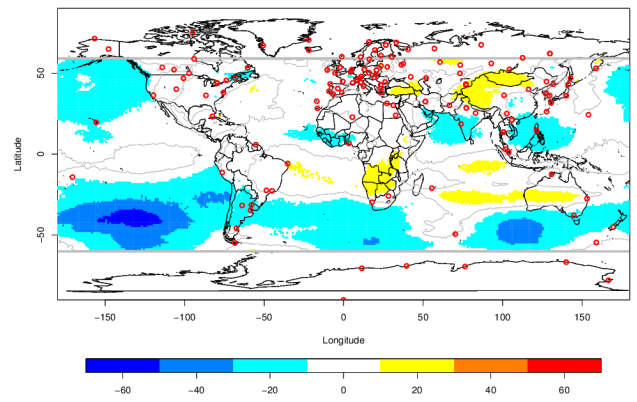

(e) SPDE (July)

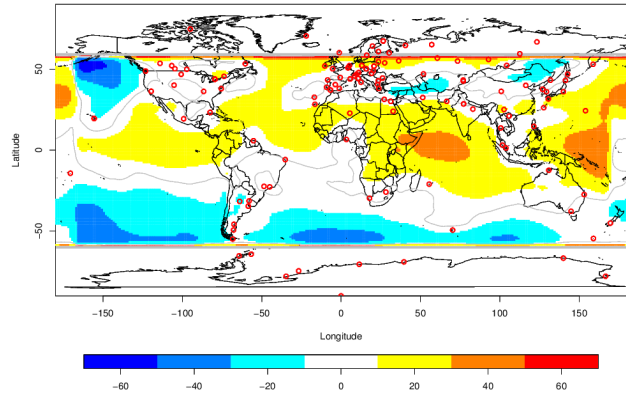

(b) CBK (January)

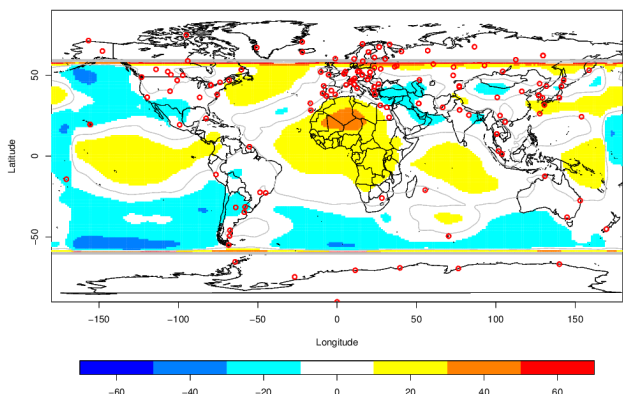

(d) CBK (April)

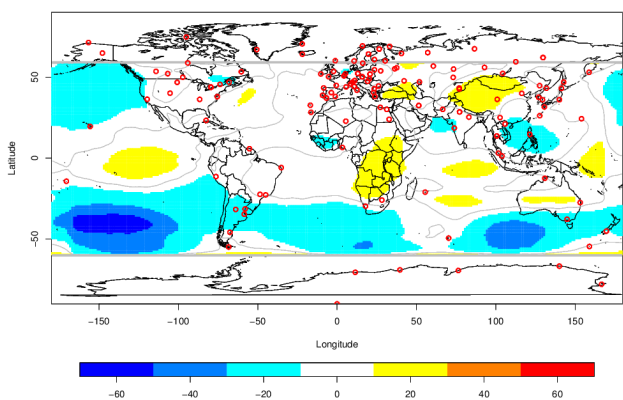

(f) CBK (July)

Figure 4. Total ozone (DU) difference mapping of SPDE and covariance-based kriging (CBK) estimated mean with respect to satellite data from January, April and July 2000.

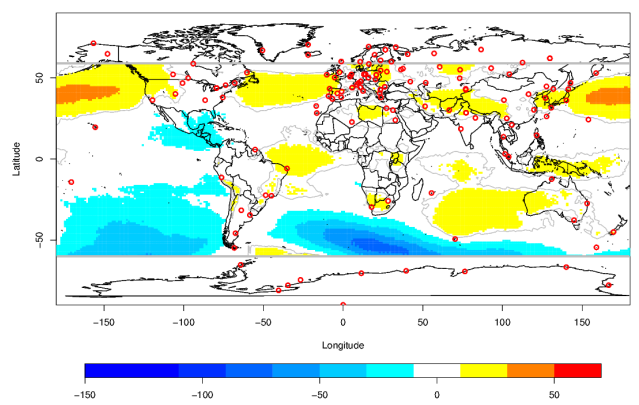

(a) SPDE (October)

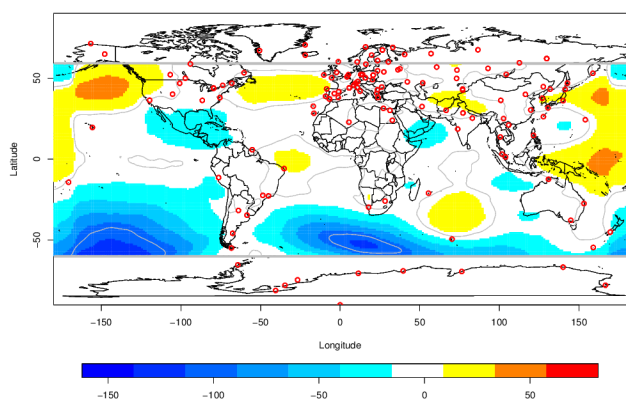

(b) CBK (October)

Figure 5. Total ozone (DU) difference mapping of SPDE and covariance-based kriging (CBK) estimated mean with respect to satellite data from October 2000. Estimation in October shows worse prediction than other months; hence it used different scale from Fig. 4. 


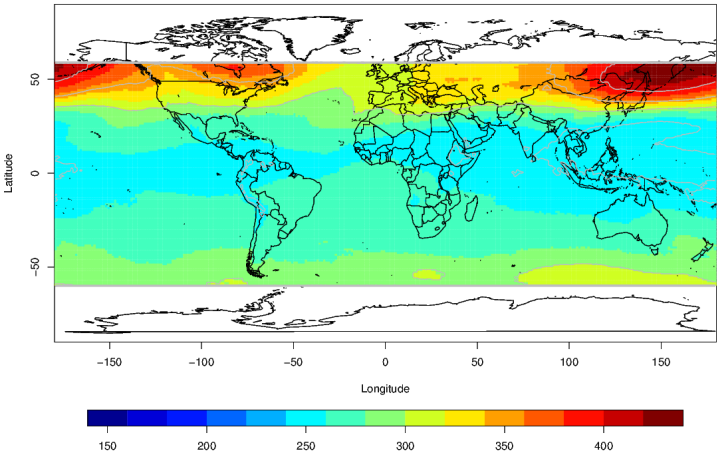

(a) TOMS DJF, 2000

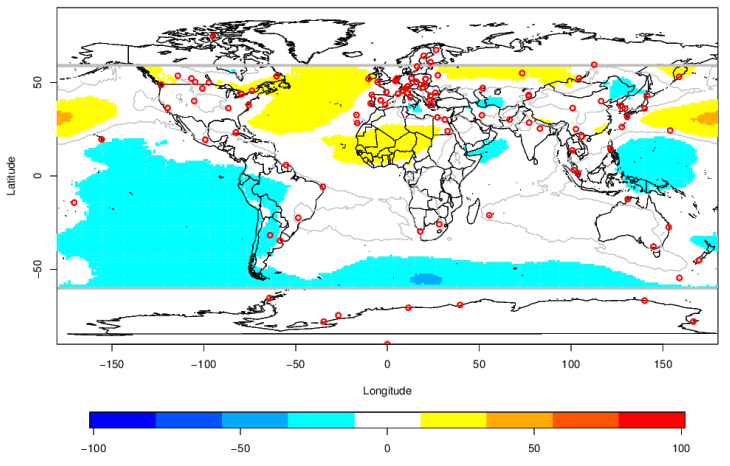

(c) SPDE (DJF)

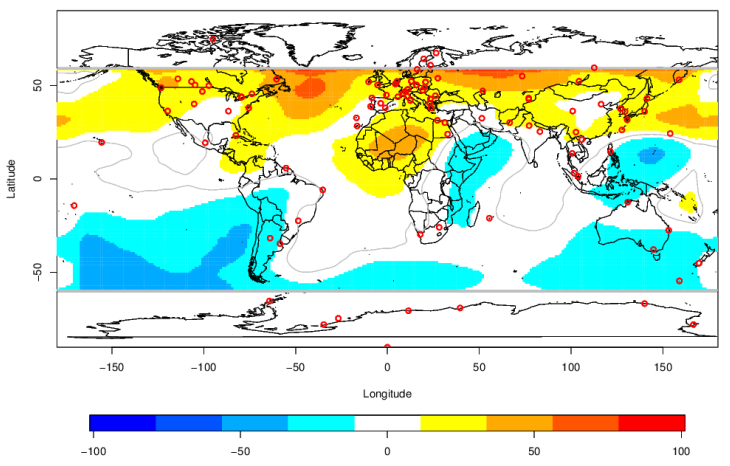

(e) $\mathrm{CBK}(\mathrm{DJF})$

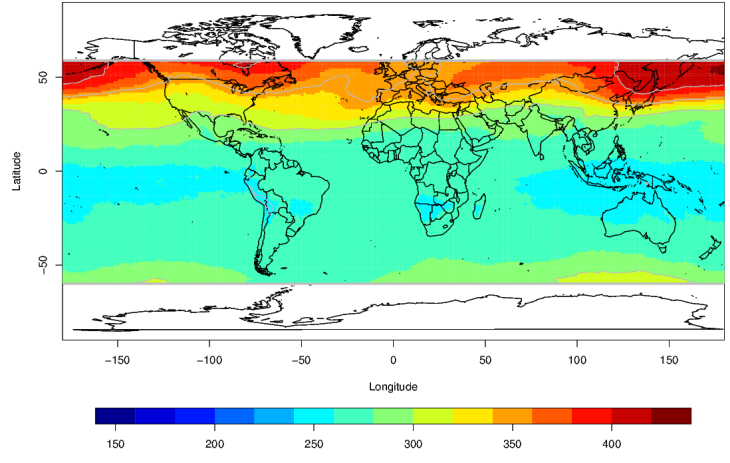

(b) TOMS MAM, 2000

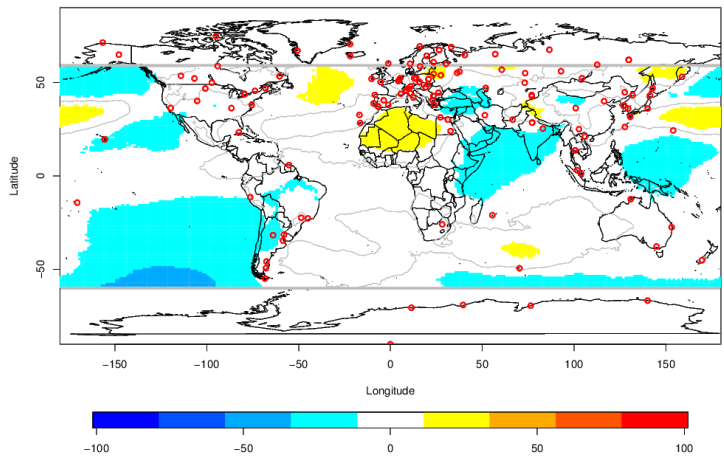

(d) SPDE (MAM)

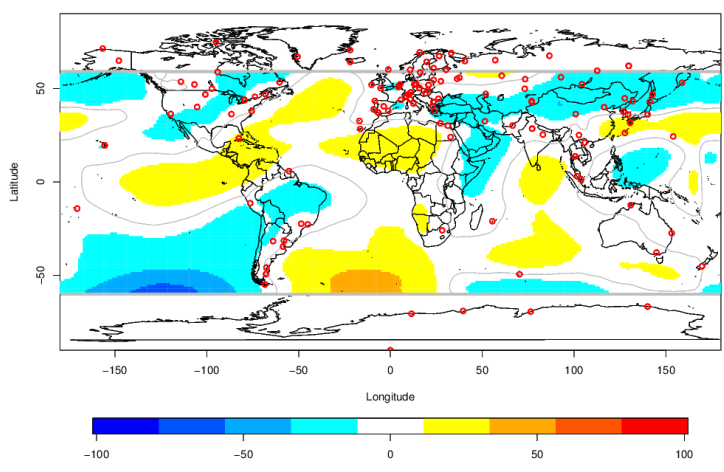

(f) CBK (MAM)

Figure 6. Ozone mapping from TOMS data in (a) DJF and (b) MAM; global difference mapping of SPDE and covariance-based kriging (CBK) predicted mean with respect to TOMS data in DJF (c and e) and MAM (d and f) 2000.

timation in particular. In those circumstances, the SPDE approach shows robustness against observations loss. Figures 6 and 7 show TOMS maps of all seasons in 2000 in top panels and differences with predictions from SPDE and covariancebased kriging. Underestimation at the South Pacific are in accordance with expectations for both techniques, but sur- face predictions by SPDE achieve a better fit than covariancebased kriging, especially in SON.

\section{Impact on long-term changes}

In this section, we show how variations in time of the zonal means can be improved by employing the more accurate 


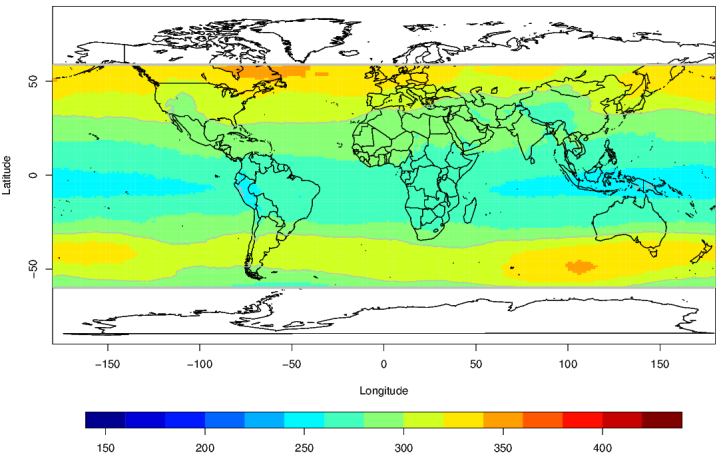

(a) TOMS JJA, 2000

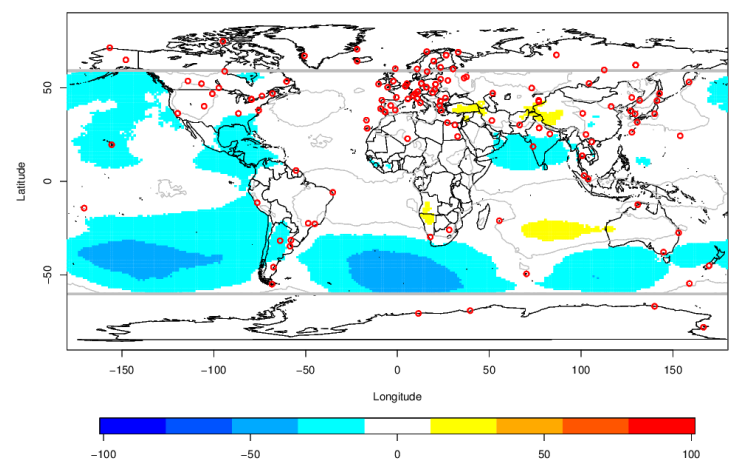

(c) SPDE (JJA)

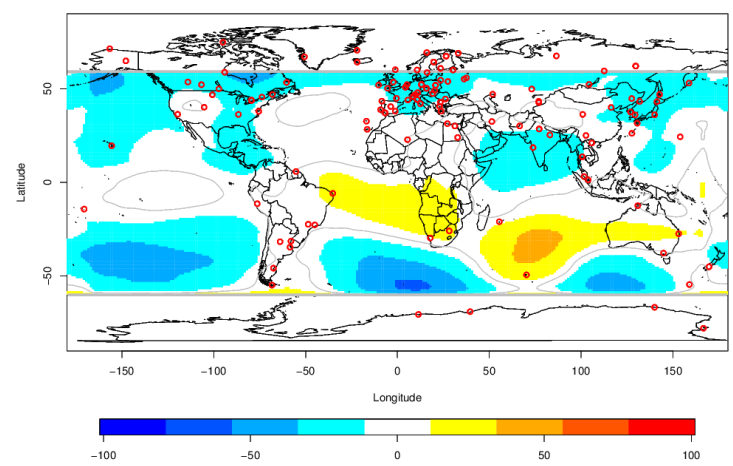

(e) CBK (JJA)

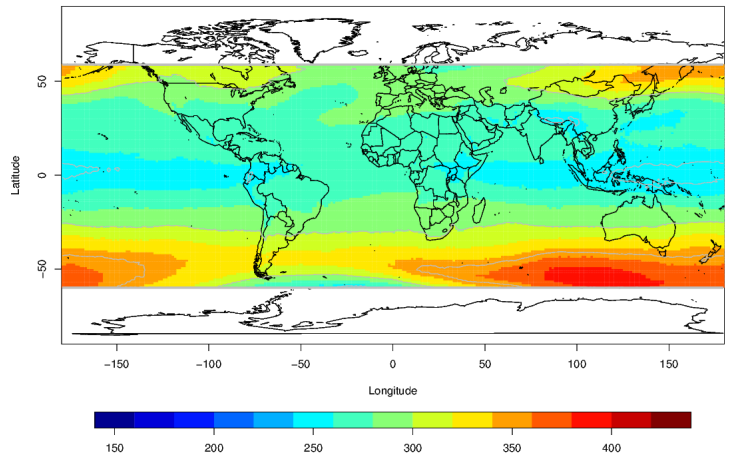

(b) TOMS SON, 2000

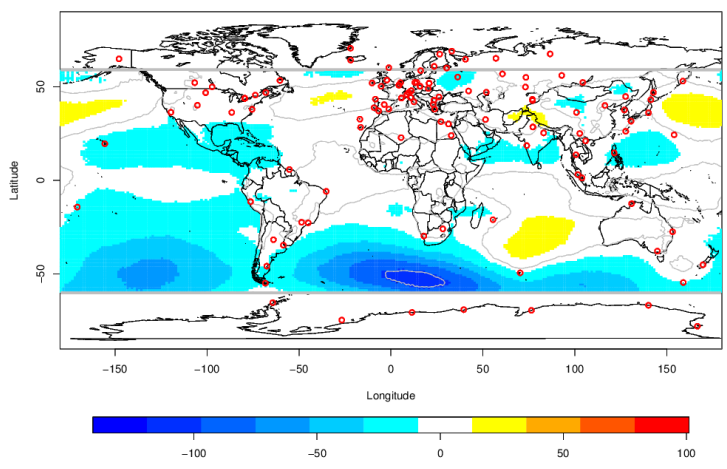

(d) SPDE (SON)

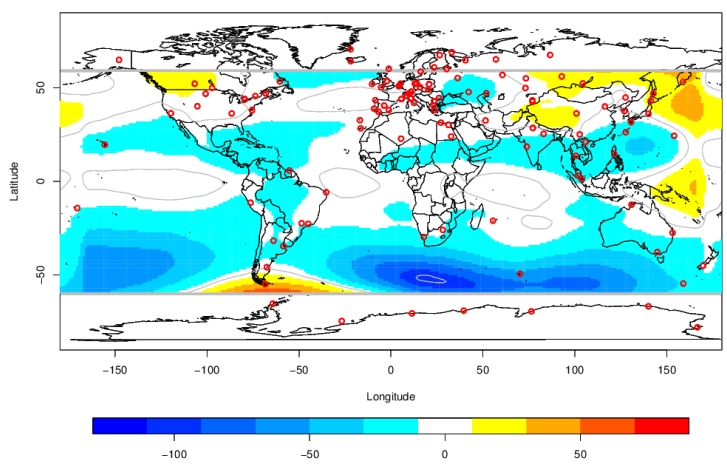

(f) $\mathrm{CBK}(\mathrm{SON})$

Figure 7. Ozone mapping from TOMS data in (a) JJA and (b) SON; global difference mapping of SPDE and covariance-based kriging (CBK) predicted mean with respect to TOMS data in JJA (c and e) and SON (d and f) 2000.

SPDE-based mapping technique instead of covariance-based kriging.

\subsection{Zonal mean time series analysis}

To see how the ozone zonal means change over time over the same stations with different algorithms, we choose the stations which supplied data for at least 25 years between 1979 and 2010. Hence 67 stations are used to construct these zonal mean time series. There is a strong asymmetry between the Southern Hemisphere (6 stations) and the Northern Hemisphere (48 stations); there are 13 stations at the tropics (defined as $\left.30^{\circ} \mathrm{S}-30^{\circ} \mathrm{N}\right)$. The zonal means were constructed by averaging the estimations obtained from either SPDE or covariance-based kriging over a grid of $1^{\circ} \times 1^{\circ}$. 
Table 4. Generalized cross-validation error $\left(\sigma_{\mathrm{GCV}}\right)$ and residual sum of square (RSS) for different strategies over 2000-2005. Monthly and seasonally results are averaged over each year, and annual results are directly estimated using annual means from each station.

\begin{tabular}{|c|c|c|c|c|c|c|c|c|}
\hline & & Year & 2000 & 2001 & 2002 & 2003 & 2004 & 2005 \\
\hline \multirow[t]{13}{*}{ Monthly } & \multirow{4}{*}{ A } & $\bar{n}$ & 143.25 & 151.58 & 147.50 & 136.17 & 135.25 & 138.42 \\
\hline & & $n_{\mathrm{eff}}$ & 34.42 & 19.20 & 35.73 & 31.49 & 20.76 & 27.65 \\
\hline & & $\sigma_{\mathrm{GCV}}$ & 12.95 & 15.39 & 12.12 & 12.71 & 14.52 & 14.13 \\
\hline & & RSS & 20681 & 33662 & 17304 & 17215 & 25838 & 23994 \\
\hline & \multirow[t]{3}{*}{ B } & $n_{\mathrm{eff}}$ & 30.95 & 17.88 & 30.82 & 24.52 & 20.51 & 25.81 \\
\hline & & $\sigma_{\mathrm{GCV}}$ & 10.85 & 12.60 & 10.34 & 10.98 & 12.75 & 11.72 \\
\hline & & RSS & 14473 & 23946 & 12693 & 13930 & 20112 & 15998 \\
\hline & \multirow[t]{3}{*}{$\mathrm{C}$} & $n_{\text {eff }}$ & 75.34 & 51.17 & 76.18 & 76.03 & 52.61 & 58.57 \\
\hline & & $\sigma_{\mathrm{GCV}}$ & 7.21 & 10.29 & 7.05 & 7.10 & 9.77 & 8.22 \\
\hline & & RSS & 4157 & 12734 & 3888 & 3019 & 9519 & 5906 \\
\hline & \multirow[t]{3}{*}{$\mathrm{D}$} & $n_{\text {eff }}$ & 81.27 & 47.06 & 80.41 & 78.16 & 55.88 & 70.26 \\
\hline & & $\sigma_{\mathrm{GCV}}$ & 6.73 & 9.48 & 6.62 & 6.66 & 8.79 & 7.64 \\
\hline & & RSS & 3306 & 10890 & 3187 & 2692 & 6923 & 4574 \\
\hline \multirow[t]{13}{*}{ Seasonally } & \multirow{4}{*}{ A } & $\bar{n}$ & 126.00 & 135.75 & 135.50 & 123.75 & 120.50 & 127.75 \\
\hline & & $n_{\mathrm{eff}}$ & 33.25 & 21.07 & 26.62 & 32.19 & 19.05 & 18.07 \\
\hline & & $\sigma_{\mathrm{GCV}}$ & 10.44 & 11.67 & 9.17 & 9.23 & 9.99 & 12.18 \\
\hline & & RSS & 9993 & 16559 & 9306 & 7630 & 10243 & 16576 \\
\hline & \multirow[t]{3}{*}{ B } & $n_{\text {eff }}$ & 28.05 & 12.48 & 24.69 & 21.11 & 18.43 & 20.82 \\
\hline & & $\sigma_{\mathrm{GCV}}$ & 8.18 & 10.38 & 7.71 & 8.37 & 8.63 & 9.63 \\
\hline & & RSS & 6533 & 15281 & 6552 & 7299 & 7781 & 9912 \\
\hline & \multirow[t]{3}{*}{$\mathrm{C}$} & $n_{\mathrm{eff}}$ & 47.87 & 48.17 & 99.78 & 69.18 & 41.10 & 44.56 \\
\hline & & $\sigma_{\mathrm{GCV}}$ & 5.23 & 8.51 & 5.58 & 5.31 & 6.78 & 7.39 \\
\hline & & RSS & 2387 & 7546 & 1025 & 1567 & 4070 & 4695 \\
\hline & \multirow[t]{3}{*}{ D } & $n_{\mathrm{eff}}$ & 63.11 & 55.65 & 84.82 & 68.48 & 51.23 & 55.28 \\
\hline & & $\sigma_{\mathrm{GCV}}$ & 5.21 & 7.29 & 4.91 & 5.48 & 6.71 & 7.15 \\
\hline & & RSS & 1888 & 5051 & 1372 & 1759 & 3507 & 3804 \\
\hline \multirow[t]{13}{*}{ Annually } & \multirow{4}{*}{ A } & $n$ & 83 & 97 & 101 & 90 & 87 & 97 \\
\hline & & $n_{\text {eff }}$ & 27.90 & 15.96 & 9.94 & 10.43 & 6.02 & 3.00 \\
\hline & & $\sigma_{\mathrm{GCV}}$ & 7.01 & 7.28 & 6.61 & 7.16 & 6.83 & 10.03 \\
\hline & & RSS & 2704 & 4301 & 3982 & 4080 & 3782 & 9452 \\
\hline & \multirow[t]{3}{*}{ B } & $n_{\mathrm{eff}}$ & 24.10 & 23.67 & 11.21 & 8.35 & 12.65 & 12.70 \\
\hline & & $\sigma_{\mathrm{GCV}}$ & 5.05 & 5.34 & 6.29 & 7.23 & 5.95 & 8.67 \\
\hline & & RSS & 1501 & 2094 & 3552 & 4271 & 2629 & 6342 \\
\hline & \multirow[t]{3}{*}{$\mathrm{C}$} & $n_{\text {eff }}$ & 47.32 & 40.50 & 48.22 & 57.58 & 25.63 & 28.00 \\
\hline & & $\sigma_{\mathrm{GCV}}$ & 3.59 & 4.56 & 4.45 & 4.54 & 5.04 & 7.65 \\
\hline & & RSS & 460 & 1172 & 1045 & 667 & 1556 & 4035 \\
\hline & \multirow[t]{3}{*}{ D } & $n_{\mathrm{eff}}$ & 56.16 & 49.55 & 49.46 & 67.83 & 36.17 & 48.02 \\
\hline & & $\sigma_{\mathrm{GCV}}$ & 3.12 & 4.08 & 4.56 & 4.93 & 5.03 & 7.11 \\
\hline & & RSS & 261 & 790 & 1076 & 539 & 1469 & 3076 \\
\hline
\end{tabular}

In order to overcome the underestimation over the South Pacific (see Fig. 4) and achieve a better estimation of longterm global zonal means, the monthly mean norms for each station were subtracted from observations over all the period. Then for each month, spatial interpolation through SPDE and covariance-based kriging were performed to the deviations.
The ozone norms were added back to these deviations in order to compare zonal means over the corresponding belts.

In this study, we compare the zonal mean time series estimated by SPDE and covariance-based kriging with Solar Backscatter Ultraviolet (SBUV) satellite instrument merged ozone data described by Frith et al. (2014) (http://acd-ext. gsfc.nasa.gov/Data_services/merged/) and a data set based 
Table 5. Comparison with satellite data over all months and averaged over 2000-2005 RMSEs for covariance-based kriging (CBK) and SPDE predictions.

\begin{tabular}{|c|c|c|c|c|c|c|c|c|c|c|c|c|c|}
\hline Month & Jan & Feb & Mar & Apr & May & Jun & Jul & Aug & Sep & Oct & Nov & Dec & Average \\
\hline CBK & 20.16 & 26.30 & 22.00 & 17.64 & 24.49 & 15.88 & 13.30 & 29.39 & 50.15 & 51.92 & 40.01 & 28.35 & 28.30 \\
\hline SPDE & 12.75 & 10.68 & 9.98 & 7.76 & 16.26 & 7.80 & 8.03 & 13.05 & 11.46 & 12.07 & 10.38 & 15.21 & 11.29 \\
\hline $\begin{array}{l}\text { Percentage of } \\
\text { improvement }\end{array}$ & 36.76 & 59.37 & 54.64 & 56.00 & 33.60 & 50.87 & 39.61 & 55.58 & 77.15 & 76.76 & 74.06 & 46.36 & 60.12 \\
\hline
\end{tabular}

Table 6. Comparison with satellite data over all seasons and averaged over 2000-2005 RMSEs for covariance-based kriging (CBK) and SPDE predictions.

\begin{tabular}{lrrrrr}
\hline Season & DJF & MAM & JJA & SON & Average \\
\hline CBK & 21.42 & 19.66 & 15.71 & 44.45 & 25.31 \\
SPDE & 18.45 & 13.54 & 8.49 & 9.20 & 12.42 \\
Percentage of improvement & 13.88 & 31.12 & 45.94 & 79.30 & 50.92 \\
\hline
\end{tabular}

on ground-based data available from the WOUDC (Bojkov and Fioletov, 1995; Fioletov et al., 2002). The SBUV merged satellite data sets incorporated the measurements from eight backscatter ultraviolet instruments (BUV on Nimbus 4, SBUV on Nimbus 7 and a series of SBUV/2 instruments on NOAA satellites) processed with the v8.6 algorithm (Bhartia et al., 2013). The WOUDC ground-based zonal mean data set is based on the following technique. Firstly, monthly means for each point of the globe were estimated from satellite TOMS data for 1978-1989. Then for each station and for each month the deviations from these means were calculated, and the belt's value for a particular month was estimated as a mean of these deviations. The calculations were done for $5^{\circ}$ broad latitudinal belts. In order to take into account various densities of the network across regions, the deviations of the stations were first averaged over 5 by $30^{\circ}$ cells, and then the belt mean was calculated by averaging these first set of averages over the belts. Until this point the data in the different $5^{\circ}$ belts were based on different stations (i.e., were considered independent). However, the differences between nearby belts are small. Hence one can reduce the errors of the belt's average estimations by using some smoothing or approximation. So the zonal means were then approximated by zonal spherical functions (Legendre polynomials cosine of the latitude) to smooth out spurious variations. This methodology (Bojkov and Fioletov, 1995) shares some ideas with SPDE in terms of taking advantage of spherical functions for spatial interpolation over the globe, but this methodology can only be conducted on zonal means calculation rather than global surface prediction. The merged satellite and the WOUDC data sets were compared again recently and demonstrated a good agreement (Chiou et al., 2014).

To investigate the pattern of zonal mean long-term changes in detail, Fig. 8 shows the monthly means from SBUV

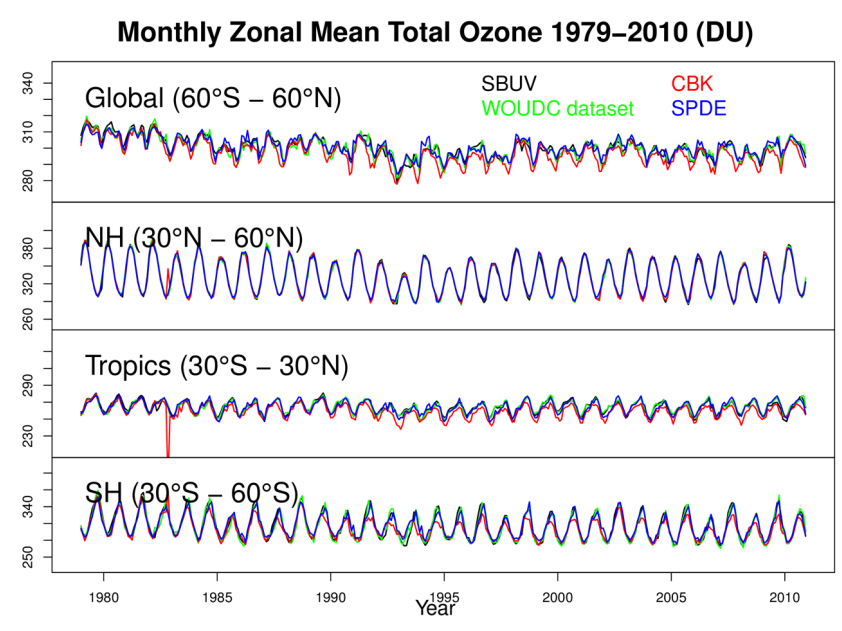

Figure 8. Time series of zonal means by SBUV satellite data (black) WOUDC data set (green), covariance-based kriging (red) and SPDE (blue) from 1979 to 2010.

merged data (black), WOUDC data set (green), SPDE (blue) and covariance-based kriging (red). SPDE and covariancebased kriging estimated means both match well satellite data and the WOUDC data set in the Northern Hemisphere. Covariance-based kriging means in the tropics fluctuate heavily and are unrealistic in some years, which indicates that covariance-based kriging may perform well at some locations but can provide distorted results at other locations; moreover the large kriging-based fluctuations in the beginning of the period may be due to a lack of stations in the early years of 1979-2010. SPDE estimated means are more robust under this circumstance. Limited stations in the South Hemisphere may trigger underestimation and deflation of the estimated annual cycle in SPDE and covariance-based kriging. Therefore we carry out a seasonal smoothing by averaging September to November to estimate better the annual peak over the Southern Hemisphere (i.e., October). This smoothing algorithm improves the match with SBUV data.

\subsection{Sensitivity analysis}

The final step is to conduct a sensitivity analysis for the long-term zonal mean estimations against either randomly removed stations or drifts in some of the ground-based observations. To see the impact of removing stations on the 

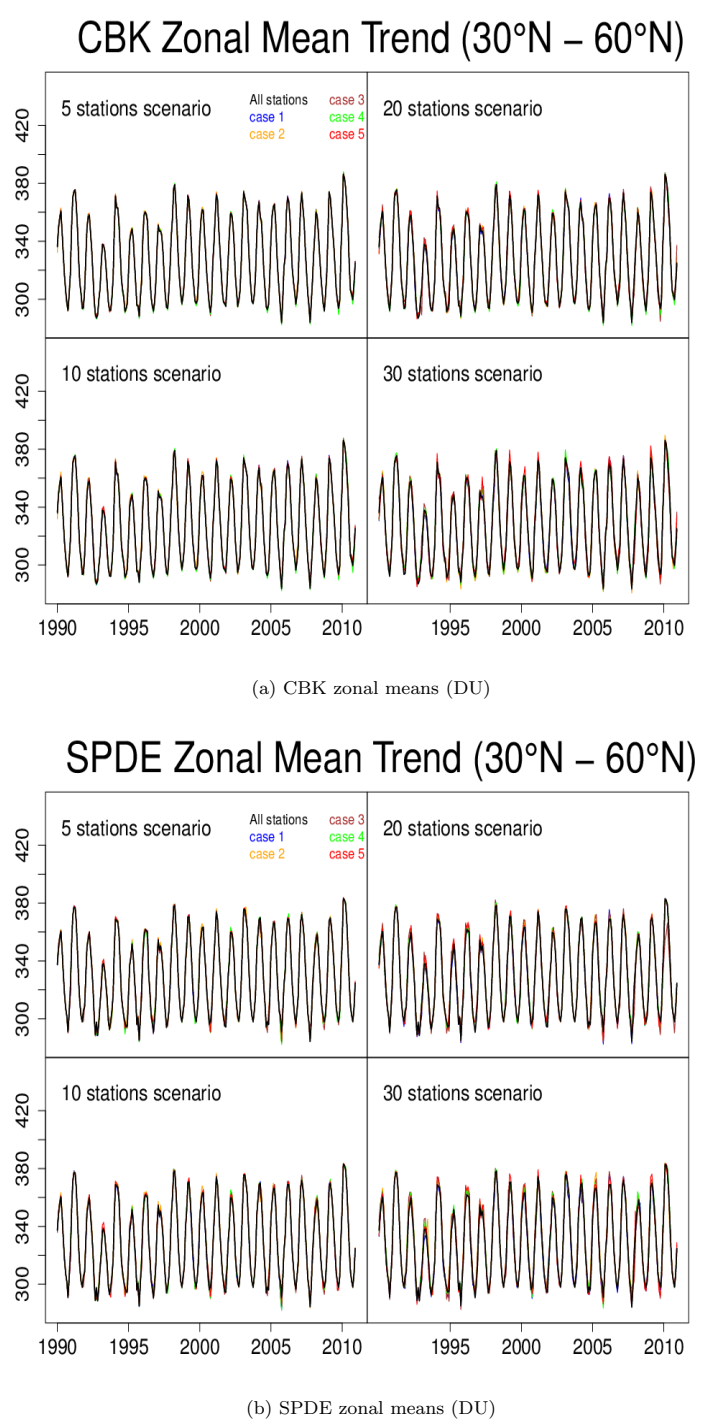

Figure 9. Time series of $30-60^{\circ} \mathrm{N}$ zonal means by (a) covariancebased kriging (CBK) and (b) SPDE from 1990 to 2010 for four scenarios with 5, 10, 20 and 30 stations removed globally including $3,6,12$ and 18 stations removed in the $\mathrm{NH}$.

long-term ozone zonal mean change, we choose 57 stations (39 stations in the Northern Hemisphere, 10 stations in the tropics and 8 stations in the Southern Hemisphere) which provided data over the entire period from 1990 to 2010 . We randomly remove 5,10, 20 and 30 stations out of these set of stations by taking into account the relative weights of the respective regions and estimate the zonal mean trends in each case. The stations removed are randomly chosen by the design in Table 7.

Furthermore, to illustrate possible variations in the sensitivity analysis, we randomly draw 5 sets of stations which need to be removed, labeled cases $1-5$. The time series for different zonal mean trends over the latitude band $30-60^{\circ} \mathrm{N}$ and $30-60^{\circ} \mathrm{S}$ are displayed in Figs. 9 and 10, respectively.
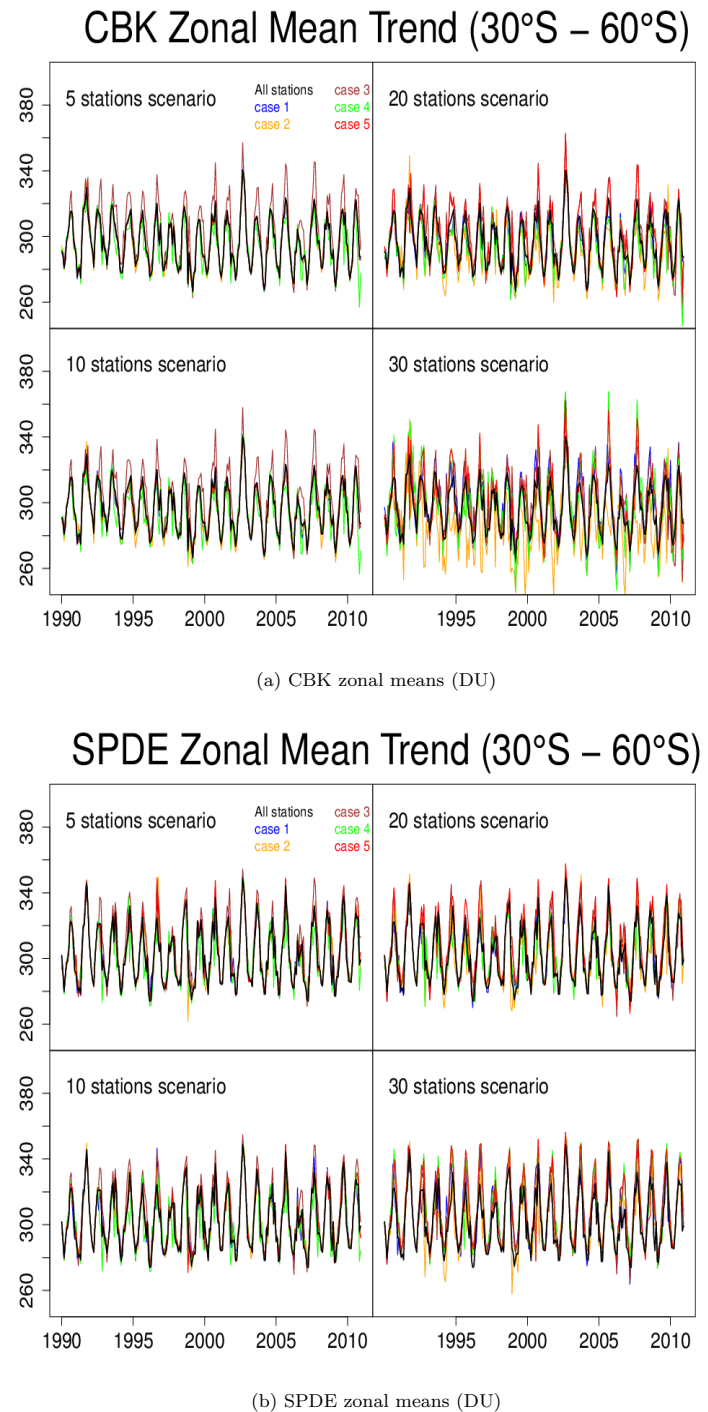

Figure 10. Time series of $30-60^{\circ} \mathrm{S}$ zonal means by (a) covariancebased kriging (CBK) and (b) SPDE from 1990 to 2010 for four scenarios with 5, 10, 20 and 30 stations removed globally including $1,2,4$ and 6 stations removed in the $\mathrm{SH}$.

The impact of randomly removing stations in the Northern Hemisphere is small even in the case of 30 stations removed (over half of the observations). The Southern Hemisphere is more sensitive to a loss of information because only few stations are located in there. The more stations are removed, the more fluctuations appears in the time series. The main finding is that the long-term effects estimated by SPDE are again more robust than the ones obtained by covariancebased kriging, especially for the case of 30 stations removed (with only two stations left in the Southern Hemisphere). The covariance-based kriging estimated trends can become chaotic for cases 2 and 4, and under this circumstance the total ozone annual cycle become unidentifiable. 


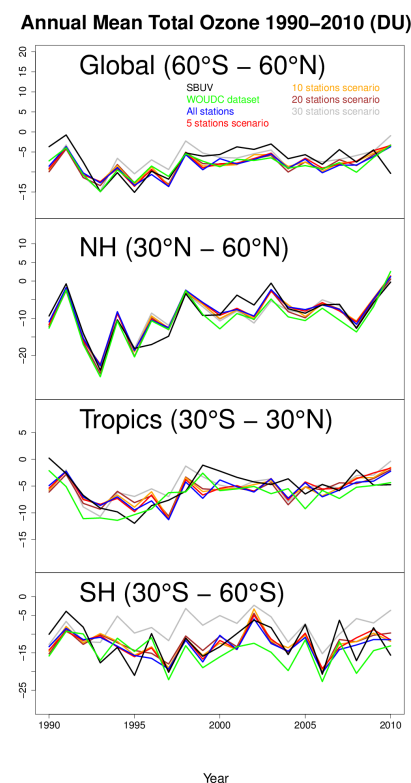

(a) SPDE annual zonal mean
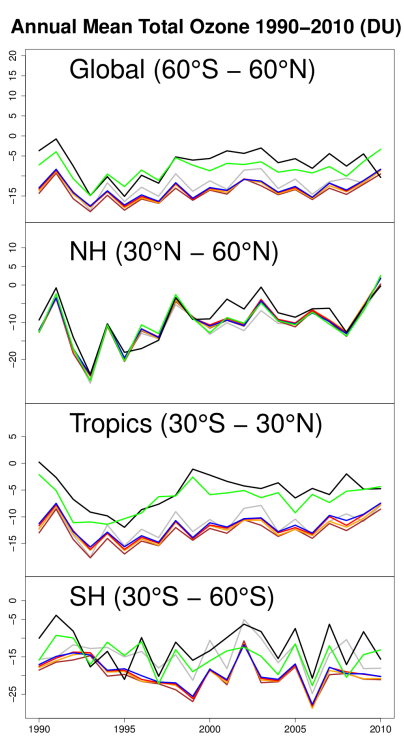

(b) CBK annual zonal mean

Figure 11. Annual zonal mean deviances from SBUV data (black), WOUDC data set (green), using all 57 available ground-based data (blue), random removed 5 (red), 10 (yellow), 20 (brown) and 30 (grey) stations in SPDE and covariance-based kriging (CBK) estimation over the (1) global $\left(60^{\circ} \mathrm{N}-60^{\circ} \mathrm{S}\right)$, (2) $\mathrm{NH}\left(30-60^{\circ} \mathrm{N}\right),(3)$ tropics $\left(30^{\circ} \mathrm{N}-30^{\circ} \mathrm{S}\right)$ and $(4) \mathrm{SH}\left(30-60^{\circ} \mathrm{S}\right)$ from 1990 to 2010.

Table 7. Design of the sensitivity analysis: stations to be removed are randomly selected within each region.

\begin{tabular}{lrrrrr}
\hline Number of removed & 5 & 10 & 20 & 30 & Total \\
\hline NH $\left(90-30^{\circ} \mathrm{N}\right)$ & 3 & 6 & 12 & 18 & 39 \\
Tropics $\left(30^{\circ} \mathrm{S}-30^{\circ} \mathrm{N}\right)$ & 1 & 2 & 4 & 6 & 10 \\
$\mathrm{SH}\left(30-90^{\circ} \mathrm{S}\right)$ & 1 & 2 & 4 & 6 & 8 \\
\hline
\end{tabular}

We use case 1 for further illustration, where both SPDE and covariance-based approaches estimated well with respect to other cases. Figure 11 shows deviations in time series in the annual mean total ozone estimated by SBUV data, WOUDC data set, SPDE and covariance-based kriging. We can see that both SPDE and covariance-based kriging estimate well in the Northern Hemisphere. Covariance-based kriging underestimates means significantly over the tropics and the Southern Hemisphere, while SPDE estimated means are close to SBUV trends. Note that SPDE estimated trends using all stations are closer to SBUV observations than the WOUDC data set at the tropics and Southern Hemisphere overall.

For the second part of sensitivity analysis, we add random long-term drifts into observations due to instrument-related problems. In reality, all observations from a ground-level station are often be biased by 5-10 DU (2-3\%) over a period of several years. For the setting of drifts, let $y_{i j}$ be the ozone ob-
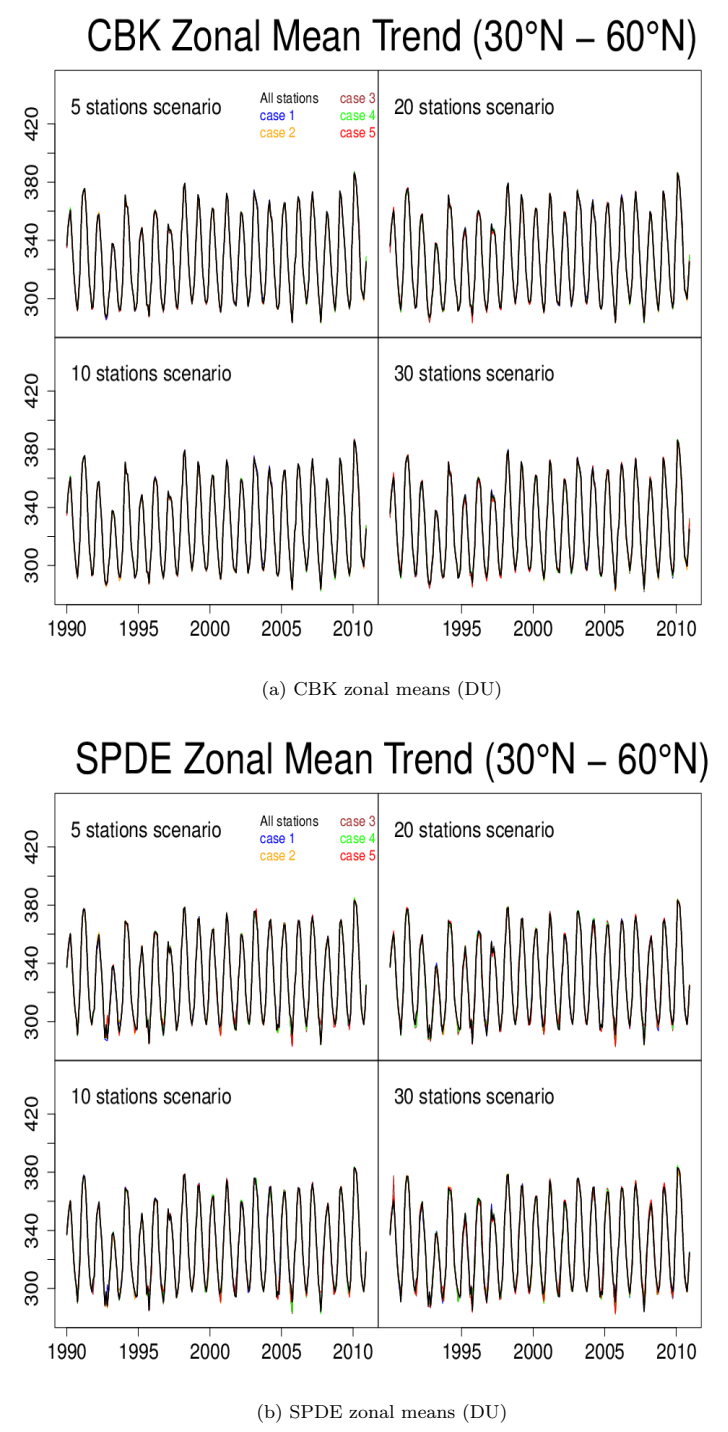

Figure 12. Time series of $30-60^{\circ} \mathrm{N}$ zonal means by (a) covariancebased kriging (CBK) and (b) SPDE from 1990 to 2010 for four scenarios with 5, 10, 20 and 30 stations drifted globally.

servations at station $i$ and time $j$. We randomly select some stations $i$ and set

$y_{i j}^{*}=a_{i} y_{i j}$,

where $a_{i} \sim N\left(1,0.03^{2}\right)$ is the slope over every 5-year periods, i.e., one slope factor for 1990-1994, then different drifts for 1995-1999, 2000-2004 and 2005-2010. This setting means that stations were randomly selected and drift values were then randomly generated, but the drifts are fixed for each particular station over every 5 or 6 years.

Using the same 57 stations which provided data consistently over 1990 to 2010, the zonal mean trends were estimated with these added drifts over subsets of randomly selected 5, 10, 20 and 30 stations. We consider five sets of random drifts as well to account for possible random vari- 

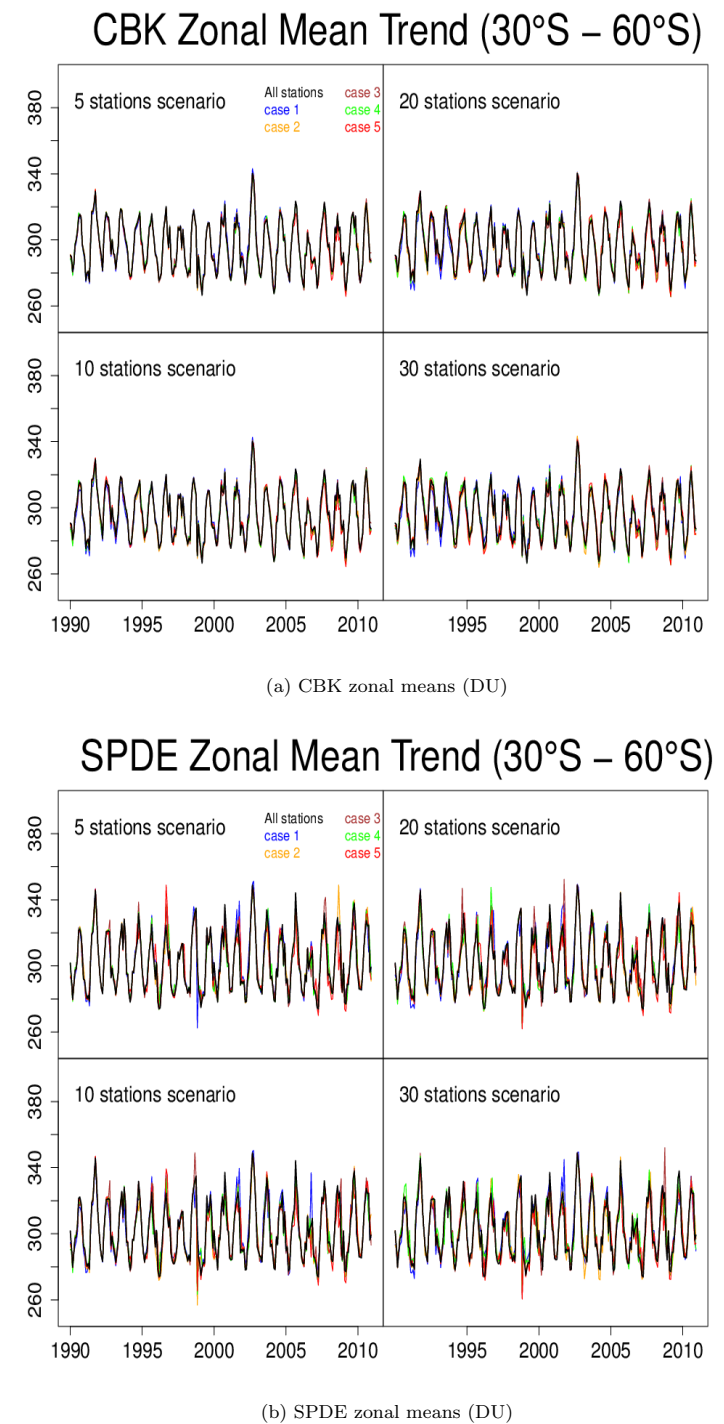

Figure 13. Time series of $30-60^{\circ} \mathrm{S}$ zonal means by (a) covariancebased kriging (CBK) and (b) SPDE from 1990 to 2010 for four scenarios with 5, 10, 20 and 30 stations drifted globally.

ations in the selection process. The time series in each case are shown in Figs. 12 and 13 for the Northern and Southern Hemisphere. Covariance-based kriging estimations hold in the case of over half of stations are drifted, SPDE also displays robustness to drift. We use case 1 as further illustration. Figure 14 shows the annual mean total ozone deviations in time series for SBUV, WOUDC data set, SPDE and covariance-based kriging estimated means when drifts are present. SPDE estimated trends turn out to be superior to covariance-based kriging over the tropics and Southern Hemisphere overall.

Table 8 reports monthly, seasonal and annual average RMSEs obtained by comparing the WOUDC, SPDE and covariance-based kriging estimated zonal means to the SBUV zonal means over 1990-2010. We can see that SPDE
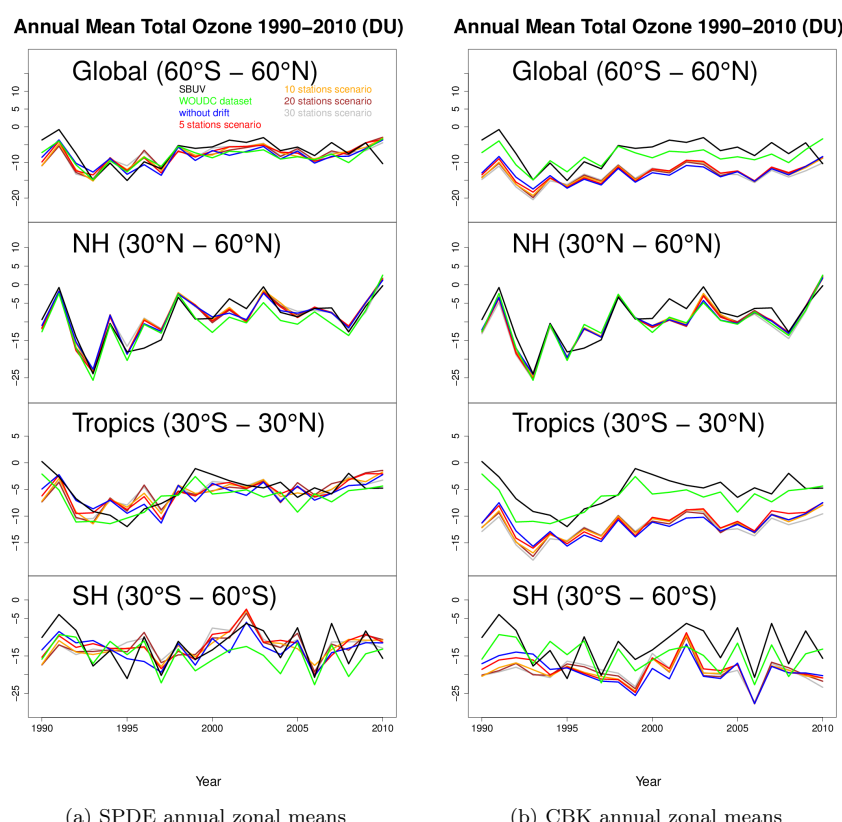

Figure 14. Annual zonal mean deviances from SBUV data (black), WOUDC data set (green), using all 57 available ground-based data (blue), adding drift to 5 (red), 10 (yellow), 20 (brown) and 30 (grey) stations in SPDE and covariance-based kriging (CBK) estimation over the (1) global $\left(60^{\circ} \mathrm{N}-60^{\circ} \mathrm{S}\right)$, (2) $\mathrm{NH}\left(30-60^{\circ} \mathrm{N}\right)$, (3) tropics $\left(30^{\circ} \mathrm{N}-30^{\circ} \mathrm{S}\right)$ and $(4) \mathrm{SH}\left(30-60^{\circ} \mathrm{S}\right)$ from 1990 to 2010.

is always superior to covariance-based kriging. Furthermore, SPDE zonal means are closer to satellite zonal means than WOUDC zonal means for annual and seasonal averages in the Northern Hemisphere, despite using fewer stations (39) than WOUDC that uses all available stations each month. It shows the remarkable ability of SPDE to interpolate variations over the globe than WOUDC. However, for monthly zonal means, SPDE zonal means are further away from satellite zonal means than WOUDC zonal means. Indeed, there is much less averaging over 1 month, and the SPDE approach can suffer from the lack of stations at some locations to describe particular monthly features that can be more pronounced than seasonal or annual averages. SPDE zonal means over the tropics and Southern Hemisphere in monthly and seasonal analysis suffer greatly as only 10 stations are used in the tropics and 8 in the Southern Hemisphere (whereas WOUDC can use up to 20-30 in the Southern Hemisphere). We expect that for operational purposes, using all the available stations (usually around $130-150$ as seen in Table 4, not 57 as done here for convenience) for each month would allow SPDE to clearly outperform WOUDC everywhere at all frequencies, as it does already with fewer stations in the Northern Hemisphere for annual and seasonal averages. Such a data set would constitute an improvement for the study of trends based on ground-level instruments. 
Table 8. RMSEs of annual, seasonal and monthly total mean ozone from WOUDC data set, and SPDE and covariance-based kriging (CBK) estimated means (using 57 stations) against SBUV data over 1990-2010.

\begin{tabular}{lrrrrrrrrr}
\hline & \multicolumn{2}{c}{ Annual } & \multicolumn{4}{c}{ Seasonal } & \multicolumn{3}{c}{ Monthly } \\
& WOUDC & SPDE & CBK & WOUDC & SPDE & CBK & WOUDC & SPDE & CBK \\
\hline NH & 3.17 & 2.37 & 2.94 & 3.40 & 3.21 & 3.54 & 3.95 & 4.21 & 4.40 \\
Tropics & 2.07 & 4.01 & 9.59 & 2.42 & 5.22 & 10.47 & 2.56 & 5.79 & 10.66 \\
SH & 4.51 & 3.96 & 8.39 & 4.39 & 8.84 & 15.33 & 6.10 & 11.07 & 15.58 \\
\hline Global & 2.28 & 2.36 & 6.85 & 2.55 & 3.55 & 8.91 & 2.92 & 4.60 & 9.03 \\
\hline
\end{tabular}

\section{Conclusions}

In summary, the covariance-based kriging method may perform fairly well globally, but displays misfit locally. The misfits will be averaged out when zonal means are estimated, but they will reveal themselves as relatively higher errors in estimations compared to the SPDE spatial prediction method for mapping. Moreover, both the estimation uncertainty of SPDE and covariance-based methods considerably depend on the location of stations, but the SPDE approach outperforms covariance-based kriging in terms of the uncertainty quantification in areas with few stations. The estimation of trends in time series over the Northern Hemisphere are more accurate for both methods than over the Southern Hemisphere as there is a much denser network of stations than in the Southern Hemisphere. The sensitivity analysis also suggests that the ground-based network can provide a reliable source of data for estimation of the long-term ozone trends. In the Northern Hemisphere, annual means can be successfully estimated even if half of the available sites is excluded from the analysis. This is not the case for the tropical belt and Southern Hemisphere where the number of sites is very limited. Additional $3 \%$ biases over 5-year intervals at up to the half of the network have a relatively small impact on the estimated zonal means. This suggest that the network can tolerate some systematic errors as long as instruments are calibrated on a regular basis ( 5 years in our tests) in order to remove such biases. Overall, when stations are removed or have added drift, the SPDE approach shows more robustness than covariance-based kriging and thus, for current observations, should be a preferred method. 


\section{Appendix A: Computational details of SPDE and covariance-based approaches}

\section{A1 SPDE approach}

The algorithm of estimation of parameters in SPDE works as follows. Let $Y(\mathbf{s})$ be an observation of the latent field $P(\mathbf{s})+$ $X(\mathbf{s})$, the model is given by

$Y(\mathbf{s})=P(\mathbf{s})+X(\mathbf{s})+\epsilon(\mathbf{s})$,

where $P$ is a polynomial which is the fixed part of the model, $X(\mathbf{s})$ is the solution of the SPDE (Eq. 3) and observation error $\epsilon(\mathbf{s})$ is zero mean Gaussian noise with variance $\sigma^{2}$. This field $X(\mathbf{s})$ can be built on a basis representation

$x(\mathbf{s})=\sum_{k=1}^{n} \psi_{k}(\mathbf{s}) w_{k}$,

where $w_{k}$ is the stochastic weight chosen so that the $x(\mathbf{s})$ approximates the distribution of solutions to the SPDE on the sphere. The basis functions $\psi_{k}(\mathbf{s})$ are chosen by a finite element method in order to obtain a Markov structure and to preserve it when conditioning on local observations. To allow an explicit expression of the precision matrix for the stochastic weights, we use a piecewise linear basis functions for the location of the observations. The overall effect of the mesh construction is that smaller triangles indicate higher accuracy of the field representation, where the observations are more dense, such as the network at the Northern Hemisphere. Larger triangles are constructed in the Southern Hemisphere as observations are more sparse, thus we can preserve computational resources. In order to balance the local accuracy and computational tractability, we add some triangles with the following restrictions. The minimum allowed distance between points is $10 / r \mathrm{~km}(r=6371$ is the Earth radius) and the maximum allowed edge length in any triangle is $500 / r \mathrm{~km}$, with the aim to refine the triangulation into an embedded spherical mesh. Let $\mathbf{C}=\left\langle\psi_{i}, \psi_{j}\right\rangle$ and $\mathbf{G}=\left\langle\nabla \psi_{i}, \nabla \psi_{j}\right\rangle$ be matrices used in the construction of the finite element solutions of the SPDE approach. Then for $\alpha=2$, the precision matrix for the weights $\left\{w_{i}\right\}$ is given by

$\mathbf{Q}=\tau^{2}\left(\kappa^{4} \mathbf{C}+2 \kappa^{2} \mathbf{G}+\mathbf{G C}^{-1} \mathbf{G}\right)$

where the elements of $\mathbf{Q}$ have explicit expressions as functions of $\kappa^{2}$ and $\tau$ (Lindgren et al., 2011).

As pointed out in Jun and Stein (2008), the spatial mean structure on a sphere can be modeled using a regression basis of spherical harmonics; however, since the data set only contains measurements from one specific event, it is not possible to identify which part of the variation in the data come from a varying mean and which part can be explained by the variance-covariance structure of the latent field. To obtain basic identifiability, the parameters $\kappa(\mathbf{s})$ and $\tau(\mathbf{s})$ are taken to be positives, and their logarithm can be decomposed as

$$
\begin{aligned}
& \log \kappa(\mathbf{s})=\sum_{k, m} \kappa_{k, m} S_{k, m}(\mathbf{s}), \\
& \log \tau(\mathbf{s})=\sum_{k, m} \tau_{k, m} S_{k, m}(\mathbf{s}),
\end{aligned}
$$

where $S_{k, m}$ is the spherical harmonic of order $k$ and mode $m$. The real spherical harmonic $S_{k, m}(s)$ of order $k \in \mathbb{N}_{0}$ and mode $m=-k, \ldots, k$ is defined as

$$
\begin{aligned}
S_{k, m}(s) & =\sqrt{\frac{2 k+1}{4 \pi} \frac{(k-|m|) !}{(k+|m|) !}} \\
& \begin{cases}\sqrt{2} \sin (m l) P_{k,|m|}(\sin L) & \text { if }-k \leq m<0, \\
P_{k, 0}(\sin L) & \text { if } m=0, \\
\sqrt{2} \cos (m l) P_{k, m}(\sin L) & \text { if } 0<m \leq k,\end{cases}
\end{aligned}
$$

where $P_{k, m}$ are associated Legendre polynomials:

$P_{k, m}(x)=(-1)^{m}\left(1-x^{2}\right)^{m / 2} \frac{d^{m}}{\mathrm{~d} x^{m}} P_{k}(x)$,

where $P_{k}$ are Legendre polynomials,

$P_{k}(x)=\frac{1}{2^{k} k !} \frac{d^{k}}{\mathrm{~d} x^{k}}\left(x^{2}-1\right)^{k}$.

Regarding the computational implementation of the SPDE approach, one common choice would be to use a MetropolisHastings algorithm, which is easy to implement but computationally inefficient (Bolin and Lindgren, 2011). A better way is to use direct numerical optimization to estimate the parameters by employing the INLA framework, available as an $\mathrm{R}$ package (http://www.r-inla.org/). The default value in $\mathrm{R}$ INLA is $\alpha=2$, but $0 \leq \alpha<2$ are also available, though yet to be completely tested (Lindgren and Rue , 2015). So with $\alpha=2$ and a spherical two-manifold, the smoothness parameter $v$ must be fixed at 1 due to the relationship $\alpha=v+d / 2$.

\section{A2 Covariance-based approach}

For the ozone data, we specified a second order linear polynomial for $P(\mathbf{s})$ and a mean zero, Gaussian stochastic process with a Matérn covariance function for $Z(\mathbf{s})$ defined in Eq. (1) and used chordal distance as spherical metric. We implemented covariance-based kriging with the $\mathrm{R}$ package fields. We also estimated the smoothness parameter $v$ as it is not fixed for the covariance-based kriging approach. 


\section{Appendix B: Model diagnostic and selection}

To assess the performance of model fitting, the residuals are considered. Raw residuals are defined as the difference of the observed values and fitted values. They can be interpreted as estimators of the errors $e_{i}$ (and are denoted by $\hat{e}_{i}$ ). Therefore the performance of model fitting can be assessed by the RSS:

$\mathrm{RSS}=\sum_{i=1}^{n} \hat{e}_{i}^{2}$

Furthermore, to choose the number of basis functions for $\kappa(s)$ and $\tau(s)$ in an SPDE and to compare the performance of SPDE and covariance-based approaches, we also used the GCV criterion (Wahba, 1985). Let $\hat{y}$ be the predictor vector for the observed $y$ with $\hat{y}=A(\lambda) y$, where $A$ is the $n \times n$ smoothing matrix, and let the $n_{\text {eff }}=\operatorname{tr} A(\lambda)$ measure the effective number of degrees of freedom attributed to the smooth surface, which is also called the effective number of parameters. The GCV criterion selects $\lambda$ as the minimizer of the GCV function:

$V(\lambda)=\frac{n^{-1}\|(I-A(\lambda) y)\|}{\left[n^{-1} \operatorname{tr}(I-A(\lambda))\right]^{2}}=\frac{1}{n} \sum_{i=1}^{n}\left(\frac{y_{i}-\hat{y}_{i}}{1-\operatorname{tr}(A(\lambda)) / n}\right)^{2}$.

In practice, the GCV function computes the weighted residual sum of squares when each data point (i.e., station) is omitted and predicted from the remaining points. The value of $\lambda$ is linked to SPDE and covariance-based kriging by minimizing the GCV function. This allows the selection of the best $\lambda$ according to fit of predictions while accounting for possible overfitting due to a large number of parameters used.

After the optimal $\lambda$ is selected, the weighted residual variance $\sigma_{\mathrm{GCV}}^{2}$ is defined as

$\sigma_{\mathrm{GCV}}^{2}=\frac{\sum_{i=i}^{n}\left(y_{i}-\hat{y}_{i}\right)^{2}}{n-\operatorname{tr}(A(\lambda))}$.

Compared to the RSS, it mitigates the effect of the number of parameters used in the model in order to provide fair comparisons of uncertainties across models. 
Acknowledgements. Kai-Lan Chang was supported by the Taiwanese government sponsorship for $\mathrm{PhD}$ overseas study. S. Guillas was partially supported by a Leverhulme Trust research fellowship on stratospheric ozone and climate change (RF/9/RFG/2010/0427).

Edited by: L. Bianco

\section{References}

Bhartia, P. K., McPeters, R. D., Flynn, L. E., Taylor, S., Kramarova, N. A., Frith, S., Fisher, B., and DeLand, M.: Solar Backscatter UV (SBUV) total ozone and profile algorithm, Atmos. Meas. Tech., 6, 2533-2548, doi:10.5194/amt-6-2533-2013, 2013.

Bojkov, R. D. and Fioletov, V. E.: Estimating the global ozone characteristics during the last 30 years, J. Geophys. Res.-Atmos., 100, 16537-16551, doi:10.1029/95JD00692, 1995.

Bolin, D. and Lindgren, F.: Spatial models generated by nested stochastic partial differential equations, with an application to global ozone mapping, Ann. Appl. Stat., 5, 523-550, doi:10.1214/10-AOAS383, 2011.

Chiou, E. W., Bhartia, P. K., McPeters, R. D., Loyola, D. G., Coldewey-Egbers, M., Fioletov, V. E., Van Roozendael, M., Spurr, R., Lerot, C., and Frith, S. M.: Comparison of profile total ozone from SBUV (v8.6) with GOME-type and ground-based total ozone for a 16-year period (1996 to 2011), Atmos. Meas. Tech., 7, 1681-1692, doi:10.5194/amt-7-1681-2014, 2014.

Fioletov, V. E., Labow, G., Evans, R., Hare, E., Köhler, U., McElroy, C., Miyagawa, K., Redondas, A., Savastiouk, V., Shalamyansky, A., Staehelin, J., Vanicek, K., Weber, M.: Performance of the ground-based total ozone network assessed using satellite data, J. Geophys. Res.-Atmos., 113, D14313, doi:10.1029/2008JD009809, 2008.
Fioletov, V. E., Bodeker, G. E., Miller, A. J., McPeters, R. D., and Stolarski, R.: Global and zonal total ozone variations estimated from ground-based and satellite measurements: 1964-2000, J. Geophys. Res., 107, ACH 21-1-ACH 21-14 doi:10.1029/2001JD001350, 2002.

Frith, S. M., Kramarova, N. A., Stolarski, R. S., McPeters, R. D., Bhartia, P. K., and Labow, G. J.: Recent changes in column ozone based on the SBUV version 8.6 merged ozone dataset, J. Geophys. Res., 119, 9735-9751, doi:10.1002/2014JD021889, 2014.

Gneiting, T.: Strictly and non-strictly positive definite functions on spheres, Bernoulli, 19, 1327-1349, doi:10.3150/12-BEJSP06, 2013.

Guinness, J. and Fuentes, M.: Covariance functions for mean square differentiable processes on spheres, submitted, 2013.

Jun, M. and Stein, M. L.: An approach to producing space-time covariance functions on spheres, Technometrics, 49, 468-479, doi:10.1198/004017007000000155, 2007.

Jun, M. and Stein, M. L.: Nonstationary covariance models for global data, Ann. Appl. Stat., 2, 1271-1289, doi:10.1214/08AOAS183, 2008.

Lindgren, F., Rue, H., and Lindström, J.: An explicit link between Gaussian fields and Gaussian Markov random fields: the stochastic partial differential equation approach, J. Roy. Stat. Soc. B, 73, 423-498, doi:10.1111/j.1467-9868.2011.00777.x, 2011.

Lindgren, F. and Rue, H.: Bayesian spatial and spatiotemporal modelling with R-INLA, J. Stat. Softw., 63, 19, 2015.

Stein, M. L.: Space-time covariance functions, J. Am. Stat. Assoc., 100, 310-321, doi:10.1198/016214504000000854, 2005.

Stein, M. L.: Spatial variation of total column ozone on a global scale, Ann. Appl. Stat., 191-210, doi:10.1214/07-AOAS106, 2007.

Wahba, G.: A comparison of GCV and GML for choosing the smoothing parameter in the generalized spline smoothing problem, Ann. Stat., 13, 1378-1402, 1985. 